\title{
The Halo Boundary of Galaxy Clusters in the SDSS
}

\author{
Eric Baxter ${ }^{1}$, Chihway Chang ${ }^{2}$, Bhuvnesh Jain ${ }^{1}$, Susmita Adhikari ${ }^{3}$, Neal Dalal ${ }^{3,4}$, Andrey Kravtsov ${ }^{2,5,6}$, Surhud More ${ }^{7}$, \\ Eduardo Rozo ${ }^{8}$, Eli Rykoff ${ }^{9,10}$, and Ravi K. Sheth ${ }^{1,11}$ \\ ${ }^{1}$ Center for Particle Cosmology, Department of Physics, University of Pennsylvania, Philadelphia, PA 19104, USA; ebax@ sas.upenn.edu \\ ${ }^{2}$ Kavli Institute for Cosmological Physics, The University of Chicago, Chicago, IL 60637, USA \\ ${ }^{3}$ Department of Astronomy, University of Illinois at Urbana-Champaign, Champaign, IL 61801, USA \\ ${ }^{4}$ Department of Physics, University of Illinois at Urbana-Champaign, Champaign, IL 61801, USA \\ ${ }^{5}$ Department of Astronomy and Astrophysics, The University of Chicago, Chicago, IL 60637, USA \\ ${ }^{6}$ Enrico Fermi Institute, The University of Chicago, Chicago, IL 60637, USA \\ ${ }^{7}$ Kavli Institute for the Physics and Mathematics of the Universe (WPI), Tokyo Institutes for Advanced Study, The University of Tokyo, 5-1-5 Kashiwanoha, \\ Kashiwa-shi, Chiba, 277-8583, Japan \\ ${ }^{8}$ Department of Physics, University of Arizona, Tucson, AZ 85721, USA \\ ${ }^{9}$ Kavli Institute for Particle Astrophysics \& Cosmology, P.O. Box 2450, Stanford University, Stanford, CA 94305, USA \\ ${ }^{10}$ SLAC National Accelerator Laboratory, Menlo Park, CA 94025, USA \\ ${ }^{11}$ The Abdus Salam International Center for Theoretical Physics, Strada Costiera 11, I-34151 Trieste, Italy \\ Received 2017 March 2; revised 2017 April 22; accepted 2017 April 26; published 2017 May 18
}

\begin{abstract}
Analytical models and simulations predict a rapid decline in the halo density profile associated with the transition from the "infalling" regime outside the halo to the "collapsed" regime within the halo. Using data from SDSS, we explore evidence for such a feature in the density profiles of galaxy clusters using several different approaches. We first estimate the steepening of the outer galaxy density profile around clusters, finding evidence for truncation of the halo profile. Next, we measure the galaxy density profile around clusters using two sets of galaxies selected on color. We find evidence of an abrupt change in galaxy colors that coincides with the location of the steepening of the density profile. Since galaxies that have completed orbits within the cluster are more likely to be quenched of star formation and thus appear redder, this abrupt change in galaxy color can be associated with the transition from single-stream to multi-stream regimes. We also use a standard model comparison approach to measure evidence for a "splashback"-like feature, but find that this approach is very sensitive to modeling assumptions. Finally, we perform measurements using an independent cluster catalog to test for potential systematic errors associated with cluster selection. We identify several avenues for future work: improved understanding of the small-scale galaxy profile, lensing measurements, identification of proxies for the halo accretion rate, and other tests. With upcoming data from the DES, KiDS, and HSC surveys, we can expect significant improvements in the study of halo boundaries.
\end{abstract}

Key words: cosmology: observations - galaxies: clusters: general

\section{Introduction}

In the standard cosmological model, gravitational collapse causes small perturbations in an initially smooth dark matter density field to collapse into dense clumps known as halos. The matter distribution in and around halos can be divided into two components, which we will refer to as "infalling" and "collapsed." Infalling material is in the process of falling toward the halo, but has not yet passed through an orbital pericenter. Such material has experienced a first turnaround at the point when gravity halted its motion away from the halo due to the expansion of the universe, but has not yet experienced a second turnaround after passing by the halo. Collapsed material, on the other hand, has experienced at least one orbital pericenter passage and is in orbit around the halo ${ }^{12}$ (Gunn \& Gott 1972; Fillmore \& Goldreich 1984; Bertschinger 1985). Close to the halo center, collapsed material dominates the mass distribution, while far away from the halo center infalling material dominates. The transition between these two regimes happens near the halo virial radius; the scale of first turnaround, on the other hand, is about five times larger.

\footnotetext{
$\overline{12}$ Note that infalling material could include collapsed subhalos that are falling into a larger halo.
}

Using $N$-body simulations, Diemer \& Kravtsov (2014, hereafter DK14) determined that stacked dark matter halo density profiles exhibit a sharp decline near the transition from the infalling regime to the collapsed regime. DK14 associated this feature with the second turnaround of dark matter particles, which results in a density caustic in the accreted material. A caustic here refers to a narrow, localized region of enhanced density; just beyond the second turnaround caustic, the density declines rapidly, producing the feature observed by DK14. Owing to the connection between the observed steepening of the profile and second turnaround, this feature has recently been termed the splashback feature. Subsequently, Adhikari et al. (2014) developed a simple model for the location of the feature and confirmed the results from DK14. The idea that second turnaround is associated with a caustic in the density profile dates back to work by Fillmore \& Goldreich (1984) and Bertschinger (1985). However, it was not obvious that a clear feature resulting from second turnaround would persist in realistic simulations and after averaging across many halos.

A significant steepening of the profile followed by a flattening as one moves outward from the center is clearly seen in the clustering signal of galaxies and clusters measured from Sloan Digital Sky Survey (SDSS) data (Abbas \& Sheth 2007; Sheldon et al. 2009) and in weak lensing measurements around SDSS clusters (Johnston et al. 2007; 
Sheldon et al. 2009). Recently, Tully (2015) presented evidence for a steep decline in the galaxy density profile and a discontinuity in the velocity dispersion around galaxy groups in a context similar to that considered here. Related investigations into cluster density profiles in the infalling and collapsed regimes using spectroscopic data have been performed by Rines et al. (2013), Gifford et al. (2017), and references therein.

More et al. (2016, hereafter M16) measured a steepening of the galaxy density profile around REDMAPPER clusters (Rozo \& Rykoff 2014; Rykoff et al. 2014) identified in data from the SDSS eighth data release (DR8; Aihara et al. 2011) and identified this steepening with the splashback feature seen by DK14. By fitting the DK14 model for the radial density profile-which accounts for the rapid steepening of the profile around the splashback radius - to their SDSS measurements, M16 determined that the model with a splashback feature provided a good fit to the data, while a model without a splashback feature did not $\left(\chi^{2}\right.$ of $60-140$ for 9 degrees of freedom). M16 then compared the location of the feature inferred from the data to expectations from $N$-body simulations, finding evidence of tension.

Identifying the splashback feature in data is challenging for many reasons. First, observers typically measure only the projected density profile of a halo rather than the $3 \mathrm{D}$ radial profile. Projection smears out the otherwise sharp splashback feature, making it harder to distinguish from a profile that does not have a splashback feature. Second, while measuring the mass profile of halos is possible with gravitational lensing, such measurements currently have relatively low signal-to-noise. Measurements of galaxy density can be used as a high signalto-noise proxy for the matter density, but doing so introduces additional uncertainties because the relation between the galaxy density and the matter density is not known precisely. Third, to increase the signal-to-noise of density profile measurements, one typically stacks measurements across halos with a range of mass, redshift, and accretion rates. Stacking can broaden the sharp splashback feature, making it more difficult to detect. Finally, effects such as halo miscentering can introduce significant systematic uncertainties into measurements of the halo profiles.

The main goal of this work is to carefully examine the transition from the infalling to collapsed regimes around galaxy cluster halos using data from SDSS. In particular, we are interested in whether the data provide evidence for a truncation of the halo density profile consistent with that seen in simulations and whether such truncation can be connected to the phase space behavior of the matter around the halo. These findings together would imply the existence a physical halo boundary. We employ the same SDSS-derived REDMAPPER cluster catalog and galaxy catalog as used in M16. The large number of REDMAPPER clusters and galaxies detected in SDSS make this data set the best currently available for measuring the galaxy density profile around clusters. We extend the modeling of M16 to include an important source of systematic error: the miscentering of halos in the cluster catalogs (George et al. 2012; Rykoff et al. 2014; Hoshino et al. 2015; van Uitert et al. 2015). Using these improved models, we explore whether the data favor the truncated Einasto model introduced by Diemer \& Kravtsov (2014) to describe the splashback feature over a pure Einasto model (Sérsic 1963; Einasto 1965). We present constraints on the steepening of the collapsed component of the halo profile near the splashback region and compare with existing literature in both data and simulations. Additionally, we investigate the relative abundance of red galaxies around the same clusters as a signature of the transition from the infalling to the collapsed regimes. Finally, we perform similar galaxy profile measurements using a cluster catalog derived from the same SDSS data but independent of the REDMAPPER cluster catalog. This test is important since it is conceivable that some feature of the REDMAPPER algorithm could lead to the appearance of an artificial splashback-like feature. Concerns about potential systematic biases affecting measurements of the splashback feature are well motivated: recent work by $\mathrm{Zu}$ et al. (2016) suggests that the quantity $\left\langle R_{\mathrm{mem}}\right\rangle$ used by M16 to split their cluster sample can be significantly contaminated by projection effects. A closer examination of the splashback feature in the absence of $\left\langle R_{\mathrm{mem}}\right\rangle$ splitting is therefore warranted.

As a brief aside, we note that the dark matter mass distribution is commonly described using the halo model (for a review, see Cooray \& Sheth 2002). In the simplest version of this model, all of the dark matter in the universe is assumed to live inside of halos. The matter distribution as measured by the halo-matter cross-correlation can then be divided into two components: the "one-halo" term, which describes the distribution of matter within halos, and the "two-halo" term, which describes the distribution of the halos themselves. In the language of the halo model, the "collapsed" material can be associated with the onehalo term and the "infalling" material can be associated with the two-halo term. Halo models can also be written down in a way that make the connection to phase space more explicit (e.g., Sheth et al. 2001). In this work, however, we will generally use the terminology of the collapsed and infalling material.

The paper is organized as follows. In Section 2, we summarize the data used in this work; in Section 3, we outline our methodology for measuring and fitting the galaxy density profiles; in Section 4, we present our findings related to constraining the halo profile in the infalling-to-collapsed transition regime; in Section 5, we present our results related to the relation between this transition and the colors of galaxies; we discuss our results and implications for future work in Section 6. Throughout this analysis, we will assume a flat$\Lambda \mathrm{CDM}$ cosmological model with $h=0.7$ and $\Omega_{M}=0.3$ and we will measure cluster-centric distances in comoving units. Logarithms in base 10 are denoted with log.

\section{Data}

We use data from SDSS in our analysis. The main data set is the same as that used by M16: the REDMAPPER galaxy clusters described in Rykoff et al. (2014) and the SDSS DR8 photometric galaxies (Aihara et al. 2011). We select galaxy clusters with richness $20<\lambda<100$ and redshifts $0.1<z<0.33$, resulting in a catalog of 8649 clusters. The photometric galaxies are selected by requiring the galaxy to have an $i$-band magnitude brighter than 21.0 (after dust extinction correction), a magnitude error smaller than 0.1 , and none of the following flags: SATURATED, SATUR_CENTER, BRIGHT, and DEBLENDED. The Landy-Szalay estimator used in Section 3.1 requires a set of random points that uniformly populate the volume of space in which clusters and galaxies could be observed. The cluster randoms used for this purpose are generated by the REDMAPPER algorithm; these incorporate the redshift and richness distribution of the REDMAPPER clusters. The galaxy randoms were generated 
by distributing points uniformly inside the footprint of the $i<21$ galaxy sample.

To select the red and blue galaxies used in Section 5, we perform an additional color cut in the rest-frame $g-r$ color. We compute this quantity using the $K$-corrected absolute magnitudes in the SDSS database, ABSMAGG and ABSMAGR. We define two subsamples: the quartile with the largest $g-r$ (the "red" sample, $g-r>0.74$ ) and the quartile with the smallest $g-r$ (the "blue" sample, $g-r<0.39$ ). For the purposes of this study, a more sophisticated selection based on, e.g., a red-sequence selection in color-magnitude-redshift space is not necessary. The simple selection defined here is sufficient to demonstrate the connection between galaxy color and features of interest in the galaxy density profile.

\section{Methods}

\subsection{Galaxy Profile Measurement}

We calculate the projected number density of galaxies around clusters, $\Sigma_{g}(R)$, as a function of the projected comoving cluster-centric distance, $R$, by cross-correlating the clusters and the galaxies. We compute the cluster-galaxy angular correlation using the Landy-Szalay estimator (Landy \& Szalay 1993) in redshift bins of $\Delta z=0.05$. We only bin the clusters in redshift bins; the galaxy photometric redshift information is not used because of the large associated uncertainties. For each redshift bin centered at $\bar{z}_{i}$, we assume that clusters with $\bar{z}_{i}-\frac{\Delta z}{2}<z<$ $\bar{z}_{i}+\frac{\Delta z}{2}$ are located at $z=\bar{z}_{i}$. We then calculate the $i$-band absolute magnitude, $M_{i}$, for all galaxies assuming that they are located at $\bar{z}_{i}$. Following M16, we then restrict the galaxy sample to $M_{i}-5 \log (h)<-19.43$, corresponding to an apparent magnitude cut of $m_{i}<21$ at the redshift limit of the cluster catalog, $z=0.33$. For each redshift bin, we measure the cluster-galaxy correlation function in 15 comoving radial bins from 0.1 to $10.0 \mathrm{~h}^{-1} \mathrm{Mpc}$. The correlation function measurements in a given redshift bin are then converted to $\Sigma_{g}$ by multiplying by the mean galaxy density in that redshift bin. Finally, we average the measurements in all redshift bins, weighting by the number of cluster-galaxy pairs in each bin. Similar to M16, we use a jackknife resampling approach with 100 subregions to estimate the covariance of our $\Sigma_{g}(R)$ measurement.

M16 also measured the galaxy density profiles around two subsamples of clusters split on the parameter $\left\langle R_{\mathrm{mem}}\right\rangle$, defined as the average of the cluster member distances from the cluster center, weighted by the probability of cluster membership. $\left\langle R_{\text {mem }}\right\rangle$ was first introduced by Miyatake et al. (2016), where it was shown that a sample of REDMAPPER clusters split on this parameter exhibited similar masses (as inferred from weak lensing observations), but different large scale clustering biases, with the larger $\left\langle R_{\mathrm{mem}}\right\rangle$ sample having a larger bias. M16 showed that the location of the splashback radius inferred from their density profile's measurements was correlated with $\left\langle R_{\mathrm{mem}}\right\rangle$. Given the connection between the splashback radius and cluster accretion rate established by DK14, it was argued that $\left\langle R_{\mathrm{mem}}\right\rangle$ could therefore provide a measure of the cluster accretion rate. However, recent work by $\mathrm{Zu}$ et al. (2016) suggests that $\left\langle R_{\mathrm{mem}}\right\rangle$ is strongly affected by projection effects, which are in turn correlated with the surrounding density field. Given these concerns, we do not rely on $\left\langle R_{\mathrm{mem}}\right\rangle$ splits in this analysis.

\subsection{Modeling the Splashback Feature}

DK14 measured the stacked density profile of dark matter halos in simulations. They fit an Einasto model (Einasto 1965; Navarro et al. 2004) to the inner halo profile (radii $r<0.5 R_{\mathrm{vir}}$, where $R_{\text {vir }}$ is the halo virial radius as defined in Bryan \& Norman (1998)) while using the relation of Gao et al. (2008) to fix the Einasto parameter $\alpha$ as a function of halo peak height, $\nu$. The peak height is defined as $\nu \equiv \delta_{c} / \sigma(M, z)$, where $\delta_{c}=1.686$ is the linear collapse threshold (which is nearly redshift and cosmology independent) and $\sigma^{2}(M, z)$ is the variance of the matter field on scales corresponding to mass $M$ at redshift $z$ (see, e.g., Gunn \& Gott 1972). Extending these fits to the outer profile $\left(r>0.5 R_{\mathrm{vir}}\right)$, DK14 found that the stacked density profiles exhibited a sharp decline relative to the Einasto fit just outside the halo virial radius; this decline was associated with the caustic produced by splashback of dark matter particles. To model this behavior, DK14 introduced simple fitting formulae. They model the halo density profile as the sum of an Einasto profile that effectively describes the collapsed material and a power-law profile that effectively describes the infalling material. ${ }^{13}$ The use of an Einasto profile to model the collapsed material is well motivated by many studies using $\mathrm{N}$-body simulations (Navarro et al. 2004, 2010; Merritt et al. 2005, 2006). The use of a power-law term to describe the infalling material is motivated by, e.g., the self-similar collapse models of Gunn \& Gott (1972). For a single peak, selfsimilar collapse models predict a power-law profile with index -1.5 . However, for CDM halos forming as a result of gravitational collapse around intially Gaussian perturbations, the infalling material is not expected to follow a pure powerlaw profile at large scales. Furthermore, nonlinear dynamics can modify the profile of infalling material within the halo. The precise form of the infalling material profile must therefore be calibrated using, e.g., $N$-body simulations. The simple powerlaw model, however, was shown to provide a good fit to the stacked profiles of simulated halos out to $\sim 9 R_{\text {vir }}$ in DK14. To model the observed steepening of the density profile near

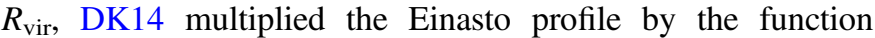
$f_{\text {trans }}(r)$, which is unity for small $r$, but declines rapidly in a narrow region near the radius $r_{t}$.

The complete profile introduced by Diemer \& Kravtsov (2014) that provides good fits to the stacked 3D density profile of simulated halos from small scales out to $\sim 9 R_{\mathrm{vir}}$ has the form

$$
\begin{gathered}
\rho(r)=\rho^{\text {coll }}(r)+\rho^{\text {infall }}(r), \\
\rho^{\text {coll }}(r)=\rho^{\operatorname{Ein}}(r) f_{\text {trans }}(r) \\
\rho^{\operatorname{Ein}}(r)=\rho_{s} \exp \left(-\frac{2}{\alpha}\left[\left(\frac{r}{r_{s}}\right)^{\alpha}-1\right]\right), \\
f_{\text {trans }}(r)=\left[1+\left(\frac{r}{r_{t}}\right)^{\beta}\right]^{-\gamma / \beta}, \\
\rho^{\text {infall }}(r)=\rho_{0}\left(\frac{r}{r_{0}}\right)^{-s_{e}},
\end{gathered}
$$

where $\rho^{\text {coll }}$ and $\rho^{\text {infall }}$ represent the profiles of the collapsed and infalling material, respectively. Note that $\rho^{\text {coll }}$ and $\rho^{\text {infall }}$

\footnotetext{
13 The DK14 model also includes a constant term equal to the mean density of the universe. Here, since the measurements are effectively mean-subtracted, we do not include such a constant term.
} 
correspond to the $\rho_{\text {inner }}$ and $\rho_{\text {outer }}$ used by DK14. Since $r_{0}$ is completely degenerate with $\rho_{0}$, we will fix $r_{0}=1.5 h^{-1} \mathrm{Mpc}$ throughout.

The profile of Equations (1)-(5) contains eight free parameters. DK14 first fit density profile measurements from simulations allowing all eight parameters to vary freely, and found that the profile provided a good fit to these measurements. Because some of the parameters in their fits were correlated, DK14 also explored how the number of free parameters could be reduced by fixing various parameter combinations. In this analysis, we will allow all eight model parameters (after fixing $r_{0}$ ) to vary independently for two reasons. First, the parameter combinations constrained by DK14 depend on quantities such as the halo peak height and the virial radius, both of which cannot be measured precisely from the data. Second, it is not necessarily true that parameter combinations that can be fixed when fitting the dark matter alone can also be fixed when fitting the galaxy distribution, given the uncertain relation between galaxies and mass. Allowing all eight parameters to vary simultaneously was also the approach taken by M16. As we will discuss below, however, allowing all eight parameters to vary freely (with some weak priors) can make it difficult to distinguish between models that have a truncation caused by $f_{\text {trans }}$ and models that have $f_{\text {trans }}=1$.

Another common parameterization for modeling the density profiles of dark matter halos is the Navarro-Frenk-White (NFW) profile of Navarro et al. (1996). The NFW profile is also known to be a good fit to simulated dark matter halos, though it may not be as successful as the Einasto model at capturing the behavior of the inner halo profile (Navarro et al. 2004, 2010; Merritt et al. 2005, 2006). Since we do not have a very strong theoretical prior to prefer the Einasto profile over the NFW profile in this analysis of galaxy density profiles, we will also consider the impact on our splashback fits of replacing the Einasto profile with the generalized NFW model (gNFW):

$$
\rho_{\mathrm{gNFW}}(r)=\frac{\rho_{i}}{\left(\frac{r}{r_{s}}\right)^{\alpha_{\mathrm{gNFW}}}\left(1+\frac{r}{r_{s}}\right)^{3-\alpha_{\mathrm{gNFW}}}},
$$

where $\rho_{i}$ sets the normalization of the profile and $\alpha_{\text {gNFW }}$ sets its shape.

Since we measure projected densities on the sky, it is necessary to integrate $\rho(r)$ along the line of sight to obtain the projected density $\Sigma(R)$ :

$$
\Sigma(R)=\int_{-h_{\max }}^{h_{\max }} d h \rho\left(\sqrt{R^{2}+h^{2}}\right),
$$

where $R$ is the projected distance to the halo center. To avoid divergence of the profiles, we restrict the line-of-sight integration to $-h_{\max }<h<h_{\max }$. We set $h_{\max }=40 h^{-1} \mathrm{Mpc}$ for the results presented here; we have found that our results are quite robust to this choice.

The above equations for $\rho(r)$ and $\Sigma(R)$ were found to accurately describe the mass distribution around simulated dark matter halos in simulations by DK14. In this work, however, we will follow M16 and apply the same models to the measured galaxy distributions, which we label with subscript " $g$ "s: $\rho_{g}(r)$ and $\Sigma_{g}(R)$ (note that these functions measure number densities rather than mass densities). That is, we are assuming that any differences between the galaxy distribution and the dark matter mass distribution (i.e., galaxy bias) can be absorbed into the fitting parameters. In the limit of constant galaxy bias, this assumption is certainly true. However, at small scales, galaxy bias is expected to be scale-dependent (e.g., Peacock \& Smith 2000; Seljak 2000) and, as a result, this assumption may break down. M16 tested this assumption using subhalo profiles around cluster-size halos in dark matter simulations, showing that it is robust. However, the galaxy density profile is not expected to follow the subhalo profile at small scales, and the precise relation between the galaxy profile and the matter profile on small scales is still an active research area (e.g., Nagai \& Kravtsov 2005; Guo et al. 2011; Budzynski et al. 2012).

In the model testing parts of this work, we will adopt an operational definition and define the splashback radius as the location of the steepest slope in the model density profiles. To differentiate between the splashback radius in the two-dimensional (2D) and three-dimensional (3D) profiles, we define $R_{\mathrm{sp}}^{3 \mathrm{D}}$ as the location of steepest slope in the three-dimensional galaxy density $\left(\rho_{g}\right)$, and $R_{\mathrm{sp}}^{2 D}$ as the analogous quantity in the projected galaxy density $\left(\Sigma_{g}\right)$. Note that alternate ways of identifying the splashback radius exist in the literature (e.g., Mansfield et al. 2016). Our definition has the benefit of being well-defined and relatively easy to measure in observational data.

\subsection{Modeling Cluster Miscentering}

To measure the cluster-centric distance $R$ in the data, we use the cluster centers computed by the REDMAPPER algorithm. REDMAPPER assigns cluster centers in a probabilistic fashion: each cluster member galaxy is assigned a probability of being the cluster center, $P_{\text {cen }}$ based on its color, magnitude, redshift, and local density (Rykoff et al. 2014). The galaxy with the highest $P_{\text {cen }}$ is then considered to be the cluster center. The model for the cluster density profile introduced in Section 3.2, on the other hand, is defined with respect to the center of dark matter halos identified in $N$-body simulations using the ROCKSTAR halo finder (Behroozi et al. 2013).

It is possible for the REDMAPPER cluster center to differ from the centers of ROCKSTAR-identified halos in two ways. First, it is possible that no cluster galaxy lies at the true center of the dark matter halo. This can happen stochastically, or if observational effects such as masking prevented the central galaxy from being observed. A second possibility is that a cluster member galaxy does lie at the true center of the dark matter halo, but it is not the galaxy with the highest $P_{\text {cen }}$. We refer to both of these effects as miscentering. Miscentering can significantly alter the measured density profile at scales below the typical miscentering distance (i.e., the distance between the assumed and true halo centers). Although the transition between the infalling and collapsed regimes occurs at scales greater than the miscentering distance, we will see below that changes to the small-scale halo profile can significantly alter how models fit the profile in the transition region. We note that M16 tested for the effects of miscentering on their determination of the splashback radius by selecting clusters with high $P_{\text {cen }}$ and repeating the density profile measurements; however, they did not include a prescription for miscentering in their model for the galaxy density.

We model the effects of miscentering following the approaches of Melchior et al. (2016) and Simet et al. (2017). The miscentered density profile, $\Sigma_{g}$, can be related to the 
profile in the absence of miscentering, $\Sigma_{g, 0}$, via

$$
\Sigma_{g}=\left(1-f_{\text {mis }}\right) \Sigma_{g, 0}(R)+f_{\text {mis }} \Sigma_{g, \text { mis }}(R),
$$

where $f_{\text {mis }}$ is the fraction of clusters that are miscentered, and $\Sigma_{g \text {,mis }}$ is the galaxy density profile for the miscentered clusters. For clusters that are miscentered by $R_{\text {mis }}$ from the true halo center, the corresponding density profile is (Yang et al. 2006; Johnston et al. 2007)

$$
\begin{aligned}
& \Sigma_{g, \mathrm{mis}}\left(R \mid R_{\mathrm{mis}}\right) \\
& \quad=\int_{0}^{2 \pi} \frac{d \theta}{2 \pi} \Sigma_{g, 0}\left(\sqrt{R^{2}+R_{\mathrm{mis}}^{2}+2 R R_{\mathrm{mis}} \cos \theta}\right) .
\end{aligned}
$$

The profile averaged across the distribution of $R_{\mathrm{mis}}$ values is then

$$
\Sigma_{g, \mathrm{mis}}(R)=\int d R_{\mathrm{mis}} P\left(R_{\mathrm{mis}}\right) \Sigma_{g, \mathrm{mis}}\left(R \mid R_{\mathrm{mis}}\right),
$$

where $P\left(R_{\text {mis }}\right)$ is the probability that a cluster is miscentered by a (comoving) distance $R_{\text {mis }}$. Following Simet et al. (2017), we assume that $P\left(R_{\text {mis }}\right)$ results from a miscentering distribution that is a 2D Gaussian on the sky. The 1D probability distribution $P\left(R_{\mathrm{mis}}\right)$ is then given by a Rayleigh distribution:

$$
P\left(R_{\mathrm{mis}}\right)=\frac{R_{\mathrm{mis}}}{\sigma_{R}^{2}} \exp \left[-\frac{R_{\mathrm{mis}}^{2}}{2 \sigma_{R}^{2}}\right],
$$

where $\sigma_{R}$ controls the width of the distribution. Following Simet et al. (2017), we set $\sigma_{R}=\tau R_{\lambda}$, where $R_{\lambda}=(\lambda / 100)^{0.2} h^{-1} \mathrm{Mpc}$ and we adopt the mean value of $\bar{R}_{\lambda}=0.98 h^{-1} \mathrm{Mpc}$ for our sample. The miscentering model is then completely specified by the parameters $f_{\text {mis }}$ and $\tau$. To determine how uncertainty on miscentering propagates to uncertainty on evidence for splashback, we will consider several different (reasonable) priors on $f_{\text {mis }}$ and $\tau$ below.

The weak lensing analysis of REDMAPPER clusters by Simet et al. (2017) assumed Gaussian priors of $f_{\text {mis }}=0.2 \pm 0.07$ and $\tau=0.4 \pm 0.1$, derived using results from Rykoff et al. (2014). Rykoff et al. (2014) quantified the miscentering of the SDSS REDMAPPER clusters based on 82 and 54 X-ray selected clusters in the XCS (Mehrtens et al. 2012) and ACCEPT (Cavagnolo et al. 2009) data sets, respectively. Follow-up studies from Hoshino et al. (2015) examined data from stripe 82 , finding that the visually determined centroid of the REDMAPPER clusters agree fairly well with the REDMAPPERdetermined centroid. Our fiducial analysis uses the Simet et al. (2017) priors, but we will also consider variations on these priors, including a model without miscentering and a model where the widths of the priors on the miscentering parameters are doubled. Existing data sets used to infer the amount of cluster miscentering are quite limited and systematics in identifying/selecting the X-ray cluster centers can introduce additional uncertainties in the inferred miscentering priors (George et al. 2012).

\subsection{Model Fitting}

We fit the model described in Sections 3.2 and 3.3 to the data using a Bayesian approach. We define a Gaussian likelihood, $\mathcal{L}$, via

$$
\ln \mathcal{L}(\boldsymbol{d} \mid \boldsymbol{\theta})=-\frac{1}{2}(\boldsymbol{d}-\boldsymbol{m}(\boldsymbol{\theta}))^{T} \boldsymbol{C}^{-1}(\boldsymbol{d}-\boldsymbol{m}(\boldsymbol{\theta})),
$$

where $\boldsymbol{d}$ is the data vector of $\Sigma_{g}$ measurements, $\boldsymbol{m}(\boldsymbol{\theta})$ is the model for these measurements evaluated at parameter values $\boldsymbol{\theta}$, and $\boldsymbol{C}$ is the covariance matrix of the data. The free parameters of the model are $\rho_{0}, \rho_{s}, r_{t}, r_{s}, \alpha, \beta, \gamma, s_{e}$, and the miscentering parameters $\tau$ and $f_{\text {mis. }}$.

To compute the posteriors on the model parameters given the likelihood of Equation (12), we run a Markov Chain Monte Carlo (MCMC) analysis using emcee (Foreman-Mackey et al. 2013). Following M16, when computing the posterior, we impose Gaussian priors on several model parameters: $\log \alpha=\log 0.2 \pm 0.6, \quad \log \beta=\log 4.0 \pm 0.2, \quad$ and $\log \gamma=$ $\log 6.0 \pm 0.2$. Note that the prior on $\alpha$ adopted here and in M16 is quite weak. The central value of $\alpha=0.2$ is motivated from $N$-body simulations: given the weak lensing mass estimates of the REDMAPPER clusters, simulations predict that dark matter halos of the same mass should have $\alpha \sim 0.2$ (Gao et al. 2008). However, what we measure is the galaxy density profile and not the dark matter profile, which is significantly less constrained. Previous measurements in SDSS and simulations by Masjedi et al. (2006) suggest that the galaxy density profile may be significantly steeper than the dark matter profile at scales smaller than $\sim 0.1$ $h^{-1}$ Mpc. Simulations also show that $\alpha$ is dependent on the halo peak height, $\nu$, which leads to a dependence on halo mass and redshift (Gao et al. 2008). Finally, Dutton \& Macciò (2014) have shown that there is significant scatter in $\alpha$ between different halos, with $\sigma_{\log \alpha}=0.16+0.03 z$. Our prior on $\alpha$ is wide enough that it has little effect on our parameter constraints. One could imagine, however, imposing a tighter prior on $\alpha$ motivated by simulations; as we will discuss below, such a prior can significantly impact model fits to the measured density profiles. The central values of the priors on $\beta$ and $\gamma$ are motivated by the analysis of DK14. Finally, we restrict $r_{s} \in[0.1,5.0] h^{-1} \mathrm{Mpc}$, $r_{t} \in[0.1,5.0] h^{-1} \mathrm{Mpc}$, and $s_{e} \in[1.0,10.0]$. Given the MCMC parameter chains, we can compute the location of the minimum of the logarithmic derivative of the $3 \mathrm{D}$ density profile, $R_{\mathrm{sp}}^{3 \mathrm{D}}$. Note that when computing $R_{\mathrm{sp}}^{3 \mathrm{D}}$, we use the profile without the modifications for miscentering since we are interested in the true splashback radius of the halo.

Throughout this analysis, we set the upper limit of the scales we fit to be $8.0 h^{-1} \mathrm{Mpc}$, since the model introduced in Section 3.2 is not expected to be a good fit much beyond $\sim 9 R_{\mathrm{vir}}$, where $R_{\mathrm{vir}}$ is the halo virial radius (DK14), and for the cluster sample considered here $R_{\mathrm{vir}} \sim 1 h^{-1} \mathrm{Mpc}$. At small scales, systematics in the galaxy density measurements are expected to become significant. This is especially important in this analysis because cluster fields are inherently crowded. Issues such as detection incompleteness, photometry inaccuracy, and blending can be important at these scales (Melchior et al. 2015, 2016). In addition, the relation between galaxy distribution and dark matter distribution become more complicated at small scales, and the model described in Section 3.2 may no longer be sufficient. In M16, a minimum scale of $0.1 h^{-1} \mathrm{Mpc}$ was used. In this analysis, we will consider two choices of the minimum scale.

Because splashback corresponds to a steepening of the outer halo density profile, it is worthwhile to consider modelindependent approaches to measuring this steepening. The logarithmic derivative of the density profile can be constrained in model-independent ways (e.g., the Savitzky-Golay approach taken by M16 and Adhikari et al. 2016). However, a steep feature in the 3D profile will appear significantly less steep in the $2 \mathrm{D}$ projected profile. Furthermore, miscentering can change 
the shape of the splashback feature in the 2D profile. These effects present a challenge for non-parametric methods, since such methods can only be applied to the measured 2D profile and cannot be used to infer the 3D profile or the profile in the absence of miscentering. Additionally, near the splashback radius, the infalling matter and collapsed matter make roughly equal contributions to the total density profile, making inferences about the collapsed material alone difficult with non-parametric methods.

\subsection{Model Comparison via the Bayesian Odds Ratio}

We first consider a model comparison approach to determine whether the data support the existence of a splashback feature. In such an approach, one must take care to define the models with and without the feature. For the model with a splashback feature, we adopt the model of DK14. Defining a model without a splashback feature is more complicated. Since $f_{\text {trans }}$ was introduced by DK14 to describe this feature, it makes sense to consider $f_{\text {trans }}=1$ as a splashback-free model as was done by M16. However, in the simulations of DK14, the Einasto parameters were fixed by fitting only the inner halo $\left(R<0.5 R_{\mathrm{vir}}\right)$. We fit the Einasto profile as part of the global fit using all radii, and in this case the steep outer profile around the splashback radius may drive the best-fit values of $r_{s}$ and $\alpha$ to smaller and larger values, respectively. This can both compromise the quality of the fit at small radii and allow the Einasto profile in the $f_{\text {trans }}=1$ model to become sufficiently steep at $R \sim R_{\mathrm{sp}}^{2 D}$ to describe steepening due to a splashback feature. For now, we will perform the model comparison between models with $f_{\text {trans }}$ free and $f_{\text {trans }}=1$, but we will return to the subtleties of this comparison in Section 4.2.

To compare the $f_{\text {trans }}=1$ and $f_{\text {trans }}=$ free models, we use a Bayesian odds ratio. The odds ratio is defined as the ratio of the posteriors for two models, $M_{1}$ and $M_{2}$, given the data $D$ and prior information $I$ (see, e.g., Ivezić et al. 2014):

$$
O_{21} \equiv \frac{p\left(M_{2} \mid D, I\right)}{p\left(M_{1} \mid D, I\right)}=\frac{P\left(D \mid M_{2}, I\right) P\left(M_{2} \mid I\right)}{P\left(D \mid M_{1}, I\right) P\left(M_{1} \mid I\right)} .
$$

Assuming we have no prior reason to prefer $M_{1}$ over $M_{2}$, the above reduces to

$$
\begin{gathered}
O_{21}=\frac{P\left(D \mid M_{2}, I\right)}{P\left(D \mid M_{1}, I\right)} \\
=\frac{\int p\left(D \mid M, \boldsymbol{\theta}_{2}, I\right) p\left(\boldsymbol{\theta}_{2} \mid M_{2}, I\right) d \boldsymbol{\theta}_{2}}{\int p\left(D \mid M, \boldsymbol{\theta}_{1}, I\right) p\left(\boldsymbol{\theta}_{1} \mid M_{1}, I\right) d \boldsymbol{\theta}_{1}},
\end{gathered}
$$

where $\boldsymbol{\theta}$ represents the parameter spaces of the two models. The terms in the numerator and denominator of Equation (14) are sometimes referred to as the evidence for $M_{1}$ and $M_{2}$, respectively. The evidence measures the probability that the data would be observed if a particular model is correct. The integrals in Equation (15) are high-dimensional, so to evaluate them we use a method that relies on the MCMC parameter chains for both models. ${ }^{14}$ To interpret the odds ratios that we compute, we use the Jeffreys' scale (Jeffreys \& Lindsay 1963). The Jeffreys' scale identifies the different regimes of $\ln \left(O_{21}\right)$ with "weak" $\left(0<\ln \left(O_{21}\right)<1.16\right)$,

\footnotetext{
$\overline{14}$ See http://www.astroml.org/book_figures/chapter5/fig_model_ comparison_mcmc.html for an example.
}

"definite" $\left(1.16<\ln \left(O_{21}\right)<2.3\right)$, "strong" $\left(2.3<\ln \left(O_{21}\right)<\right.$ $4.6)$, and "very strong" $\left(4.6<\ln \left(O_{21}\right)\right)$ preference for one model over another. In addition to the odds ratio, we also report the $\Delta \chi^{2}$ between the two models in order to compare with M16.

\subsection{Test with the Alternative Cluster Catalog}

The construction of a cluster catalog like REDMAPPER requires many non-trivial choices that can potentially lead to poorly understood selection effects that could alter the measured $\Sigma_{g}$. To make any claim of a splashback detection more robust, it is therefore important to test the measurements on alternative cluster catalogs. A large number of clusterfinding algorithms exist in the literature (van Breukelen \& Clewley 2009; Soares-Santos et al. 2011; Wen et al. 2012; Rykoff et al. 2014).

To this end, we repeat the measurements of $\Sigma_{g}$ using the group catalog of Yang et al. (2007, hereafter Y07). The Y07 catalog contains groups of galaxies identified in SDSS DR7 data across a wide range of masses, including clusters as massive as the REDMAPPER clusters. Unlike the REDMAPPER algorithm, the $\mathrm{Y} 07$ algorithm does not require grouping galaxies in color space (see Yang et al. 2005 for a detailed description of the algorithm). Instead, the Y07 algorithm uses spectroscopic redshift information to iteratively assign galaxies to groups. Briefly, this iterative assignment assumes that all groups live in dark matter halos whose masses are estimated from an assumed mass-to-light relationship. Given the estimated halo mass, galaxy assignment is performed assuming that the galaxy distribution is described by an NFW profile. The halo centers and the mass-to-light ratio are then adjusted and group assignment is iterated until convergence is achieved. The scatter in the halo masses, $M_{h}$, assigned in this fashion is expected to be roughly twice as large as the scatter in the mass estimates obtained from the REDMAPPER mass-richness relation. $^{15}$

We select groups in the Y07 catalog with halo masses in the range of $13.90<\log \left(M_{h} / M_{\odot}\right)+\log h<14.89$. Accounting for the different mass definitions used by $\mathrm{Y} 07$, this mass range corresponds roughly to the mass of the $20<\lambda<100$ clusters in REDMAPPER, assuming the mass-richness relation derived in Simet et al. (2017). We impose a redshift selection of $0.1<z<0.2$, where the upper cutoff comes from Y07 and the lower bound is to match the REDMAPPER catalog. The redshift range is slightly different from the REDMAPPER cluster sample, but we do not expect significant redshift-dependences of the splashback feature resulting from this difference. This selection yields a total of 3292 groups, which is roughly 2.5 times fewer than in the REDMAPPER cluster sample. This is mainly due to the different redshift ranges of the two samples.

We measure the $\Sigma_{g}$ profile around the Y07 groups using the galaxy sample described in Section 2 and fit these measurements using the models described in Section 3.2. For the miscentering model, since $f_{\text {mis }}$ and $\tau$ are not well constrained for the Y07 catalog, we adopt the loose miscentering priors of Model D in Table 1.

\footnotetext{
15 The most massive groups in Y07 are estimated to have a mass scatter of $\sim 0.2$ dex. Using the Simet et al. (2017) mass-richness relation, the REDMAPPER mass scatter is expected to be roughly $\sim 0.1 \mathrm{dex}$, or half that of the Y07 mass estimates.
} 
Table 1

Results of Model Comparison with Various Modeling and Data Choices

\begin{tabular}{|c|c|c|c|c|c|}
\hline Model & Catalog & Priors & Scales $\left[h^{-1} \mathrm{Mpc}\right]$ & $\Delta \chi^{2}$ & $\overline{\ln \left(O_{21}\right)}$ \\
\hline A: no miscentering & $\mathrm{RM}$ & $f_{\text {mis }}=0.0, \tau=0.0$ & $0.1<R<8.0$ & 139 & 69 \\
\hline B: fixed miscentering & $\mathrm{RM}$ & $f_{\mathrm{mis}}=0.2, \tau=0.4$ & $0.1<R<8.0$ & 73.3 & 36 \\
\hline C: fiducial miscentering & $\mathrm{RM}$ & $f_{\mathrm{mis}}=0.2 \pm 0.07, \tau=0.4 \pm 0.1$ & $0.1<R<8.0$ & 5.2 & 8.9 \\
\hline D: wide miscentering prior & $\mathrm{RM}$ & $f_{\mathrm{mis}}=0.2 \pm 0.14, \tau=0.4 \pm 0.2$ & $0.1<R<8.0$ & 2.6 & 3.2 \\
\hline E: excluding small scales & $\mathrm{RM}$ & $f_{\mathrm{mis}}=0.2 \pm 0.07, \tau=0.4 \pm 0.1$ & $0.3<R<8.0$ & 0.8 & 0.6 \\
\hline F: NFW profile & $\mathrm{RM}$ & $f_{\mathrm{mis}}=0.2 \pm 0.07, \tau=0.4 \pm 0.1$ & $0.1<R<8.0$ & 42.8 & 31 \\
\hline G: wide miscentering prior, tighter prior on $\alpha$ & Y07 & $f_{\mathrm{mis}}=0.2 \pm 0.14, \tau=0.4 \pm 0.2, \log \alpha=\log (0.2) \pm 0.1$ & $0.1<R<8.0$ & 14.1 & 7.8 \\
\hline
\end{tabular}

Note. RM indicates the REDMAPPER catalog, Y07 indicates the catalog of Y07. $\Delta \chi^{2}$ and $\ln \left(O_{21}\right)$ values indicate the results of the model comparison between the $f_{\text {trans }}=1$ and $f_{\text {trans }}=$ free models, and are computed as described in Section 3.5.

\section{Evidence for Halo Truncation}

\subsection{Model Comparison Tests and Halo Miscentering}

The projected galaxy density profile, $\Sigma_{g}(R)$, measured around REDMAPPER clusters is shown as the blue points with error bars in Figure 1. Our measurement appears to be in excellent agreement with that of M16. We now explore various model fits to these measurements and the results of our model comparison tests.

We first consider as a check on our measurements the case where miscentering is not included in the model and we fit the data over a range of scales from 0.1 to $8.0 \mathrm{~h}^{-1} \mathrm{Mpc}$. This is essentially identical to the analysis of M16. The inferred $\Delta \chi^{2}$ and Bayesian odds ratio between the models with $f_{\text {trans }}=1$ and $f_{\text {trans }}=$ free are listed in Table 1 (REDMAPPER Model A). In this case, we find significant preference for the model with $f_{\text {trans }}=$ free, with $\Delta \chi^{2} \sim 139$ relative to the $f_{\text {trans }}=1$ model. This value is consistent with the $\Delta \chi^{2}$ reported by M16. The evidence ratio also indicates strong preference for the $f_{\text {trans }}=$ free model, having a value of $\ln O_{21} \sim 76$, amounting to "decisive" evidence on Jeffreys' scale. The best-fit model parameters for the two models are shown in Table 2. Also listed in Table 2 are the constraints on $R_{\mathrm{sp}}^{3 \mathrm{D}}$ determined from the $f_{\text {trans }}=$ free model fits. We note that our determination of $R_{\mathrm{sp}}^{3 \mathrm{D}}$ is consistent with that of M16. We do not list $R_{\mathrm{sp}}^{2 D}$ here, but note that it is smaller than $R_{\mathrm{sp}}^{3 \mathrm{D}}$ due to projection.

Next, we consider how miscentering affects the preference for the $f_{\text {trans }}=$ free model. We first explore the case where the miscentering parameters $f_{\text {mis }}$ and $\tau$ are fixed to the central values from the priors of Simet et al. (2017; Model B in Table 1). In this case, the $\Delta \chi^{2}$ and the evidence ratio are decreased significantly relative to the no-miscentering model, though both still indicate strong preference for the $f_{\text {trans }}=$ free model. Comparing the Models A and B for REDMAPPER in Table 2, we see that including miscentering in the model fits changes the best-fit $r_{s}$ under the model with $f_{\text {trans }}=$ free from $0.85 h^{-1} \mathrm{Mpc}$ to $0.32 h^{-1} \mathrm{Mpc}$.

When we allow the miscentering parameters to vary while imposing the priors from Simet et al. (2017; Model C), we find that the $\Delta \chi^{2}$ and odds ratio are reduced even further. As shown in Table 2 , the $f_{\text {trans }}=1$ model fits the data in this case with a large miscentering fraction, $f_{\text {mis }}=0.47$. This value is disfavored at roughly $3 \sigma$ by the miscentering priors of Simet et al. (2017). As we discuss below, we find that it is precisely the miscentering prior that is largely driving the $f_{\text {trans }}=1$ model to be disfavored.

To help understand how miscentering is affecting the fits to $\Sigma_{g}(R)$ and the results of the model comparison, we show the model fits with the Simet et al. (2017) miscentering priors (Model C) in Figure 1. The left panels of the figure illustrate the fits with $f_{\text {trans }}=1$, while the right panels illustrate the fits when $f_{\text {trans }}=$ free (note that in the left panel, the green and red curves are identical since $f_{\text {trans }}=1$ ). Comparing the gray curves (which represent the total model without miscentering) to the black dashed curves (which represent the total model with the preferred miscentering), we see that miscentering has the effect of flattening the inner galaxy profile of the clusters. This makes sense: the offsets caused by miscentering mean that the density profile is effectively averaged across scales on the order of the miscentering radius, causing an otherwise sharp inner profile to be flattened. This redistribution of the density at small scales also has the effect of narrowing the minimum in the logarithmic derivative of the profile.

Figure 1 makes it clear that the models with $f_{\text {trans }}=$ free and with $f_{\text {trans }}=1$ can both fit the data very well (as further evidenced by the low value of $\Delta \chi^{2}$ in this case). The residuals for both fits (shown in the bottom panels of the figure) appear almost identical between the two model fits. The preference for one model over the other, then, is driven by the priors on the model parameters, in particular, the prior on $f_{\text {mis }}$, as we will discuss below. The minimum of the logarithmic derivative of $\Sigma_{g}$ occurs in roughly the same location in both fits, as can be seen in the middle panels of Figure 1. However, while the two models generate very similar total profiles (and similar total logarithmic derivatives), they fit the data in significantly different ways. To see this, consider the red curves in Figure 1, which show the profile of the collapsed component when $f_{\text {trans }}=1$ (left) and when $f_{\text {trans }}=$ free (right). We see that the $f_{\text {trans }}=1$ model fits the outer profile $\left(R \gtrsim 0.5 h^{-1} \mathrm{Mpc}\right)$ with a large value of $\alpha$. In general, larger $\alpha$ results in a shallower inner profile for $r<r_{s}$. However, in the case of the $f_{\text {trans }}=1 \mathrm{fit}$, the value of $r_{s}$ is decreased, which results in a steep profile at $R \lesssim 0.5 h^{-1} \mathrm{Mpc}$; a large $f_{\text {mis }}$ then flattens the inner profile somewhat. The model with $f_{\text {trans }}=$ free, on the other hand, prefers a shallower inner profile at the same radii (as a result of larger $r_{s}$ ), does not require as much miscentering, and is steepened substantially by the $f_{\text {trans }}$ term at $R \gtrsim 0.5 h^{-1} \mathrm{Mpc}$.

The model with $f_{\text {trans }}=1$ prefers a miscentering fraction of $f_{\text {mis }} \sim 0.45$, in tension with the miscentering prior, which prefers $f_{\text {mis }}=0.2$. This tension between the preferred 

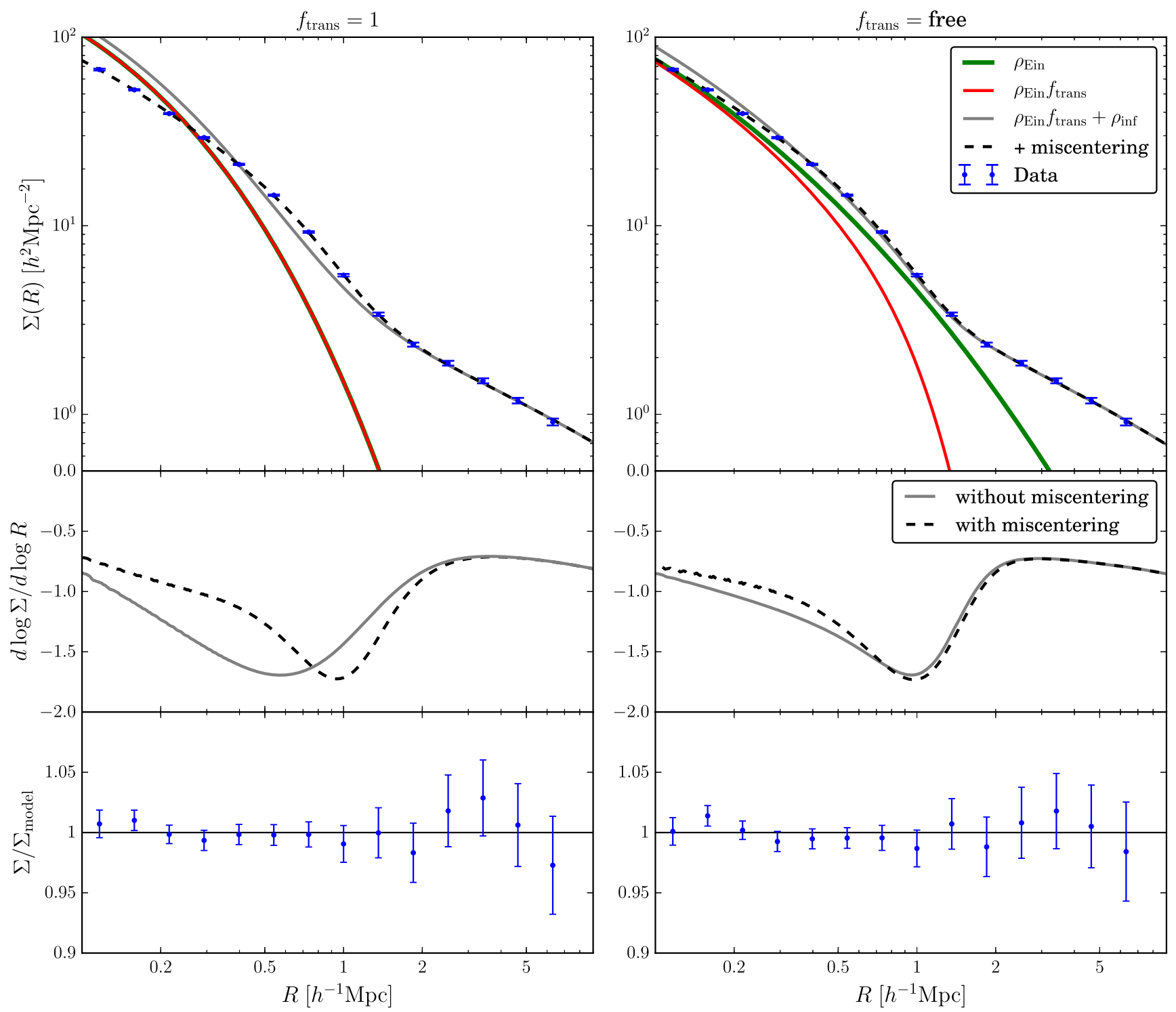

Figure 1. Measured galaxy profile $\Sigma_{g}$ around REDMAPPER clusters in SDSS and the corresponding best-fitting models. The top panels show $\Sigma_{g}$ measurements and model fits, the middle panels show the logarithmic derivative of the $\Sigma_{g}$ models, and the bottom panels show the ratio of the $\Sigma_{g}$ data points to the model. The left panels shows the model fits with no steepening function (i.e., $f_{\text {trans }}=1$ ), while the right panels show the fits with additional steepening beyond an Einasto profile (i.e., $f_{\text {trans }}$ is allowed to vary). The red curves in the upper panels show contribution to the projected galaxy density from the collapsed component $\left(\rho_{g}^{\text {coll }}(r)=\rho^{\text {Ein }}(r) f_{\text {trans }}(r)\right)$. The green curve in the right panel shows the contribution from the Einasto term of the model $\left(\rho^{\mathrm{Ein}}(r)\right)$. The gray curves are the total profile without miscentering, while the dashed black curves are the profiles with miscentering. Comparing the left and the right panels reveals that a model with large miscentering and $f_{\text {trans }}=1$ can produce a very similar total profile to a model with small miscentering and $f_{\text {trans }}$ free.

miscentering fraction and the miscentering prior, when combined with the behavior of the Einasto profile, drives the preference for the model with $f_{\text {trans }}=$ free evaluated using the evidence ratio. In support of this conclusion, when we allow more freedom in the miscentering model by doubling the widths of the Simet et al. (2017) miscentering priors (Model D in Table 1), we find that the evidence ratio in favor of $f_{\text {trans }}=$ free is weakened by roughly a factor of 300 relative to the case with Model $\mathrm{C}$ miscentering priors. Solely by going from a model without miscentering (Model A) to a model with weak miscentering (Model D), the log odds ratio has been reduced from $\ln O_{21}=69$ to $\ln O_{21}=3.2$. We note, though, that the odds ratio on the Jeffreys' scale is still "strong," even with these weaker miscentering priors. When we explore the extreme case of no priors on the miscentering parameters, we find that the odds ratio is reduced to $\ln O_{21}=0.86$, amounting to only "weak evidence."

As noted previously, M16 also considered the effects of miscentering on their analysis, but took a very different approach than that taken here. M16 repeated their measurements of the galaxy density using only clusters of low miscentering probability $\left(P_{\text {cen }}>0.9\right)$, finding that the change in $R_{\mathrm{sp}}^{3 \mathrm{D}}$ was within measurement uncertainty. We have repeated this test using our measurements, finding similar results. However, we do find that the galaxy density profile for the high $P_{\text {cen }}$ clusters is somewhat steeper on small scales than for the full sample, as is expected for a sample with better centering. While miscentering may not significantly impact the location of 
Table 2

Best-fit Model Parameters with $f_{\text {trans }}$ Free (Number Preceding Semicolon in Each Column) and $f_{\text {trans }}=1$ (Number Following Semicolon) and Under Different Modeling Assumptions

\begin{tabular}{|c|c|c|c|c|c|c|c|c|c|}
\hline Model & Catalog & $r_{s}\left[h^{-1} \mathrm{Mpc}\right]$ & $r_{t}\left[h^{-1} \mathrm{Mpc}\right]$ & $\alpha$ & $\bar{\beta}$ & $\gamma$ & $\overline{f_{\text {mis }}}$ & $\overline{\tau \tau}$ & $R_{\mathrm{sp}}^{3 \mathrm{D}}\left[h^{-1} \mathrm{Mpc}\right.$ \\
\hline A & RM & $0.85 ; 0.36$ & $1.25 ;-$ & $0.10 ; 0.42$ & $3.83 ;-$ & $6.26 ;-$ & $0.0 ; 0.0$ & $-;-$ & $1.23 \pm 0.05$ \\
\hline B & $\mathrm{RM}$ & $0.32 ; 0.29$ & $1.31 ;-$ & $0.16 ; 0.41$ & $3.71 ;-$ & $6.42 ;-$ & $0.22 ; 0.22$ & $0.32 ; 0.32$ & $1.16 \pm 0.05$ \\
\hline $\mathrm{C}$ & $\mathrm{RM}$ & $0.27 ; 0.20$ & $1.38 ;-$ & $0.17 ; 0.41$ & $3.98 ;-$ & $6.73 ;-$ & $0.22 ; 0.47$ & $0.34 ; 0.40$ & $1.18 \pm 0.08$ \\
\hline E & $\mathrm{RM}$ & $0.35 ; 0.44$ & $1.34 ;-$ & $0.23 ; 0.93$ & $3.66 ;-$ & $6.45 ;-$ & $0.20 ; 0.22$ & $0.42 ; 0.43$ & $1.15 \pm 0.07$ \\
\hline $\mathrm{F}$ & $\mathrm{RM}$ & $0.79 ; 0.10$ & $1.23 ;-$ & $1.54 ; 0.74$ & $3.65 ;-$ & $6.23 ;-$ & $0.21 ; 0.50$ & $0.45 ; 0.33$ & $1.22 \pm 0.17$ \\
\hline
\end{tabular}

Note. Modeling choices are described in Table 1. We have excluded some parameters in this table for clarity. The remaining parameters are given in Table 3.

$R_{\mathrm{sp}}^{3 \mathrm{D}}$, as we have shown above, it can still have a significant impact on the inferred model parameters and the shape of the logarithmic derivative of the profile. Furthermore, the $P_{\text {cen }}$ parameter in REDMAPPER does not fully encapsulate all possible mechanisms of cluster miscentering, such as the intrinsic scatter between the center of the dark matter halo and the BCG (since the REDMAPPER center is constrained to be on top of one of the cluster galaxies). For these reasons, it is important to include miscentering when modeling the halo profile as we have done here.

Given the impact of systematics such as miscentering, nonlinear galaxy bias, detection incompleteness, photometry inaccuracy, and blending on the inner density profile, it makes sense to consider removing the innermost scales when fitting the galaxy density measurements. We perform such a fit by excluding scales below $0.3 h^{-1} \mathrm{Mpc}$; the results are shown as Model E in Tables 1 and 2. We find that when scales below 0.3 $h^{-1} \mathrm{Mpc}$ are excluded, the data no longer exhibit a statistically significant preference for $f_{\text {trans }} \neq 1: \Delta \chi^{2}=0.8$ and odds ratio of 1.8. This is not surprising given that this is effectively ignoring all the constraining power on small scales, which we have seen previously is very important for distinguishing the $f_{\text {trans }}=1$ and $f_{\text {trans }}=$ free models.

The above analysis highlights the fact that allowing additional freedom in the inner galaxy density profile significantly affects the ability of the data to distinguish between models with $f_{\text {trans }}=1$ and $f_{\text {trans }} \neq 1$. This behavior can be understood in the following way. The Einasto model of Equation (2) couples the inner profile and the outer profile: as $\alpha$ is increased, the inner profile becomes shallower while the outer profile becomes steeper. If one ignores miscentering in the modeling of the galaxy density profile (as was done in M16), then the value of $\alpha$ is strongly constrained by the inner profile to be $\alpha \sim 0.2$. In this case, fitting the data at intermediate scales requires truncation of the Einasto profile by the $f_{\text {trans }}$ term, and $f_{\text {trans }}=1$ will be excluded at high significance. If, on the other hand, one allows for miscentering (or removes the innermost scales), the inner density profile can be fit by larger $\alpha$, smaller $r_{s}$, and larger $f_{\text {mis. }}$. Since a model with larger $\alpha$ already has a steep outer profile, the preference for additional steepening in the outer halo profile (as parameterized with $f_{\text {trans }}$ ) is reduced. We compare the posteriors on $\alpha, r_{s}$, and $f_{\text {mis }}$ for the two model fits in Figure 2 .

We note that the high values of $\alpha$ preferred by the model fits with $f_{\text {trans }}=1$ are disfavored by other studies. As shown in Table 2, these fits generally prefer $\alpha$ in the range of $0.3-0.4$. These values are well within our prior of

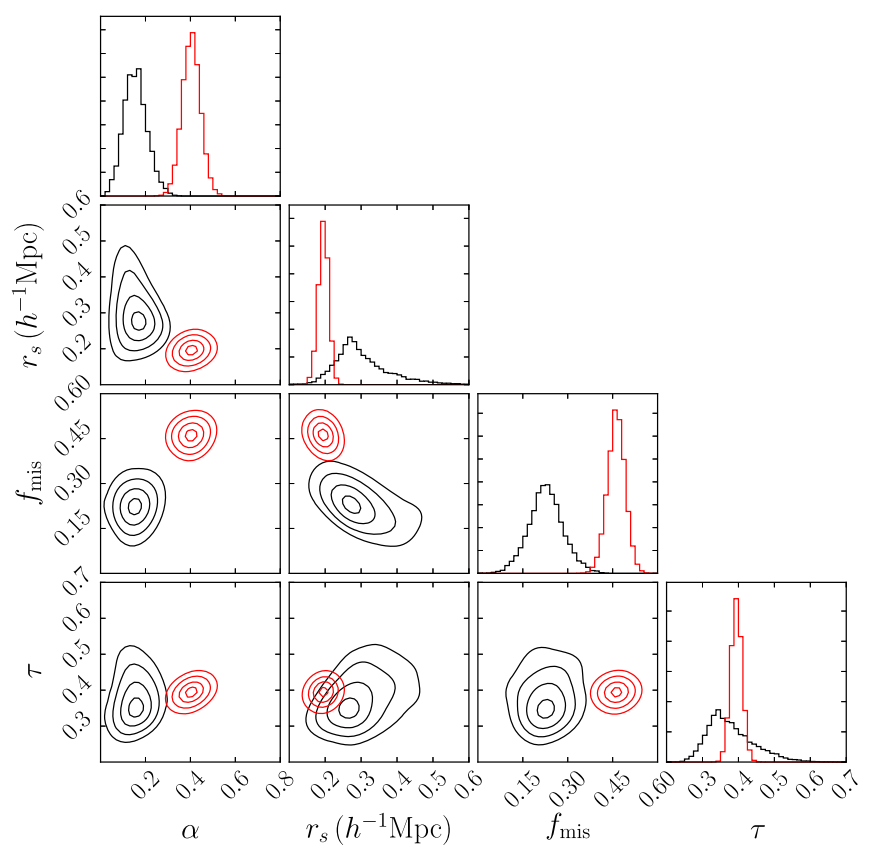

Figure 2. Posteriors on the galaxy profile parameters recovered from the MCMC analysis of the galaxy profile measurements. Black curves show results of the analysis that allow the parameters in the $f_{\text {trans }}$ term of Equation (4) to be free, while red curve shows results when $f_{\text {trans }}=1$. Both analyses use the Model C miscentering priors from Table 1.

$\log \alpha=\log 0.2 \pm 0.6$, but may be somewhat extreme relative to expectations for the dark matter from $N$-body simulations (Gao et al. 2008). A tighter prior on $\alpha$ from a combination of simulations and data would mean less sensitivity to the uncertainties in the miscentering parameters, and would therefore help improve our ability to make a more robust case for $f_{\text {trans }} \neq 1$ using the model comparison approach explored in this section.

Unlike the Einasto profile, the gNFW profile of Equation (6) forces the slope of the outer halo profile to asymptote to -3 at large radius, regardless of parameter values. Therefore, we expect that if the measured outer halo profile exhibits a sharp steepening, the preference for $f_{\text {trans }} \neq 1$ will be greater when assuming a gNFW profile than when using the Einasto model. Indeed, as shown in Table 1 (Model F), the evidence for $f_{\text {trans }}=1$ is increased when using the gNFW model. In some sense, the gNFW analysis provides a better measure of the 


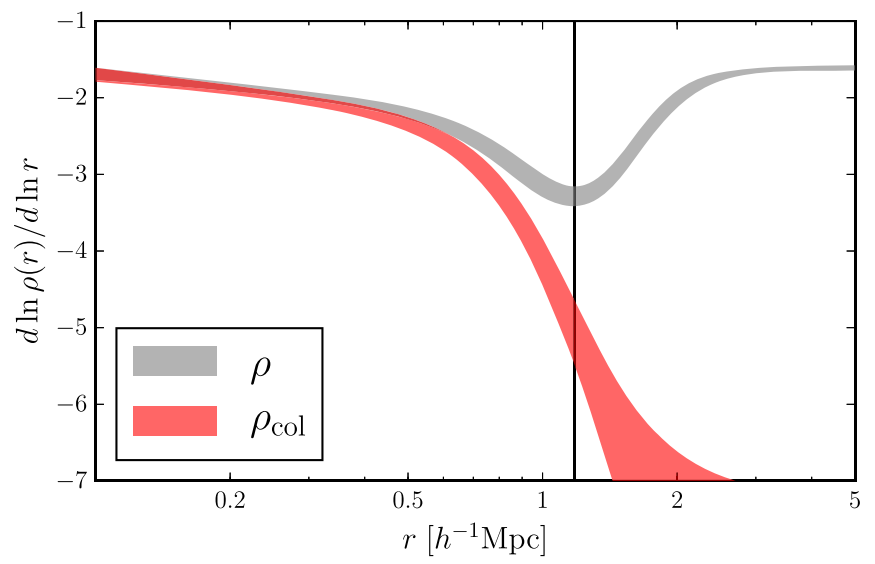

Figure 3. Constraints on the 3D logarithmic derivative of the collapsed component $\left(\rho_{g}^{\text {coll }}(r)\right)$ and total galaxy density $\left(\rho_{g}(r)\right)$ from our model fits to the measured galaxy density profile around REDMAPPER clusters. The best-fit value of the splashback radius, $R_{\mathrm{sp}}^{3 \mathrm{D}}$, is shown as the vertical line. The data prefer a profile that exhibits a steepening to slopes significantly steeper than -3 over a narrow range in radius. This finding can be interpreted as evidence for truncation of the halo profile consistent with that seen in simulations by DK14.

detection significance of $f_{\text {trans }} \neq 1$ because the outer slope is essentially fixed. However, the sensitivity of the model comparison results to the parameterization of the profile of the collapsed component is certainly a drawback to this approach to detecting a splashback-like feature.

\subsection{The Slope of the Halo Profile near $R_{s p}$}

We have seen that once miscentering is introduced into the $f_{\text {trans }}=1$ model from DK14, the resultant model is flexible enough to produce a steep outer profile while still being consistent (at moderate significance) with our fiducial priors. Consequently, the ability of the data to distinguish between this model and the $f_{\text {trans }}=$ free model is reduced. An alternative to the model comparison approach is to use the model fits to directly constrain the logarithmic derivative of the halo profile in the infalling-to-collapsed transition regime, as shown in Figure 3. Using simple collapse models, Dalal et al. (2010) argued the power-law index of the outer halo profile is set by the profile of the initial perturbation that gave rise to the halo. For power-law initial profiles of arbitrary steepness, the steepest logarithmic slope of the collapsed outer halo profile is -3 . Departures from the power-law form can give rise to logarithmic slopes slightly steeper than -3 , and for an NFW profile the slope asymptotes to -3 at large radii. Finding that the halo profile reaches logarithmic slopes significantly steeper than -3 over a narrow range of radii, then, would imply truncation of the halo profile. ${ }^{16}$

To constrain the logarithmic slope of the galaxy density profile, we draw sample profiles from our MCMC chains for the fits with $f_{\text {trans }}=$ free and compute the logarithmic derivatives of these profiles. Figure 3 shows the resultant constraints on the logarithmic derivatives of $\rho_{g}(r)$ (gray band) and $\rho_{g}^{\text {coll }}(r)$ (red band). The figure shows that we obtain a fairly tight constraint on the slope of $\rho_{g}^{\text {coll }}$ out to radii at least as large as $R_{\mathrm{sp}}^{3 \mathrm{D}}$ (i.e., where the logarithmic derivative of the

\footnotetext{
${ }^{16}$ Halos that are simulated into the future and left to relax exhibit truncated Hernquist profiles (Hernquist 1990) that reach slopes of -4 ; however, this steepening happens gradually rather than over a narrow region around $R_{\mathrm{sp}}$ (Busha et al. 2005).
}
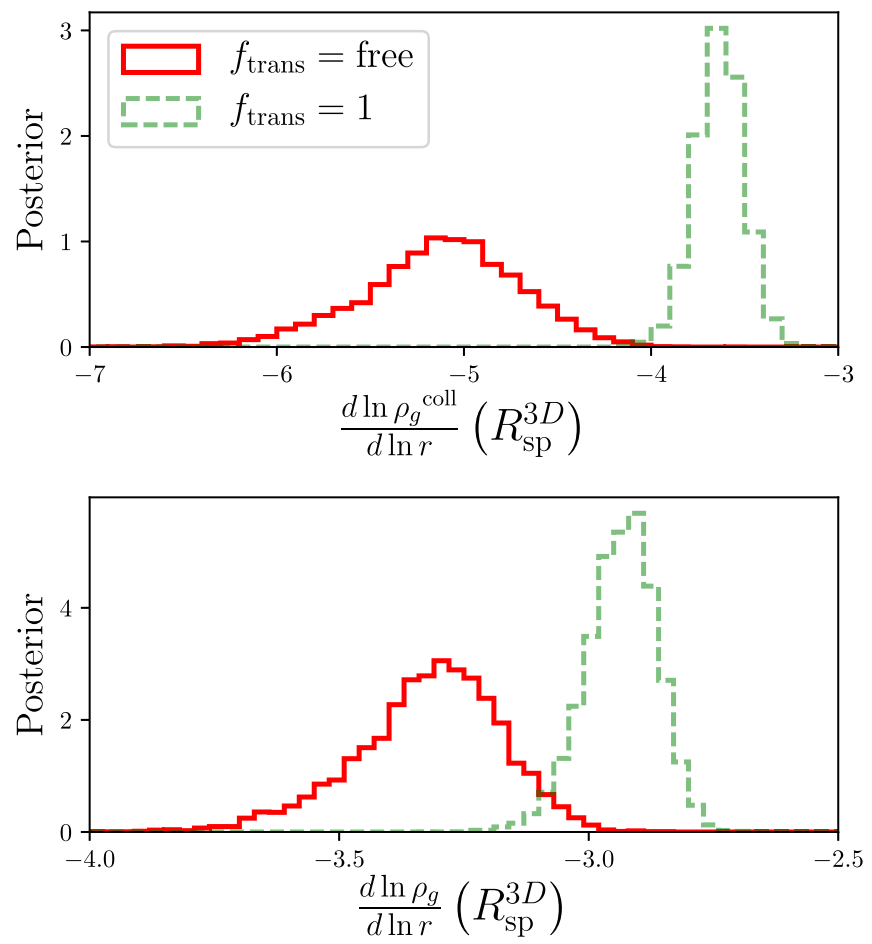

Figure 4. Constraint on the logarithmic derivative of the three-dimensional profile of collapsed component (top panel) and the total galaxy profile (bottom panel) evaluated at the splashback radius, $R_{\mathrm{sp}}^{3 \mathrm{D}}$, inferred from model fits to the measured galaxy density profiles. Solid (red) curves show results for fits when the parameters describing the profile of the splashback feature (i.e., $f_{\text {trans }}$ ) are allowed to vary, while dashed (green) curves show results for fits with $f_{\text {trans }}=1$. As discussed in the text, the $f_{\text {trans }}=$ free model provides a better description of the data. We find significant evidence for slopes of the collapsed material profile significantly steeper than -3 at the splashback radius, suggesting a truncation of the halo profile.

total profile has a minimum, marked with a vertical line in the figure). This is a non-trivial finding since $\rho_{g}^{\text {coll }}$ and $\rho_{g}^{\text {infall }}$ have roughly equal magnitudes in this regime and therefore make degenerate contributions to the total profile. It is clear from Figure 3 that over a narrow range of radius (roughly $0.7 h^{-1} \mathrm{Mpc}$ to $1.3 h^{-1} \mathrm{Mpc}$ ) the profile of the collapsed component exhibts a rapid steepening from logarithmic slopes shallower than -3 to logarithmic slopes steeper than -5 . This rapid steepening to slopes less than -3 can be taken as evidence for truncation of the halo profile.

The use of the model fits to infer the logarithmic derivative of the galaxy density profile is well motivated. We have shown that these models provide excellent fits to the data (as illustrated by the residuals in Figure 1) and the parameterization used here is known to be a good fit to the splashback feature in simulations (DK14). Additionally, M16 has shown using simulations that this approach can be used to accurately recover the 3D profile from the projected $2 \mathrm{D}$ profile. Finally, given the considerable freedom we have allowed in our model fits, we expect our constraints on the logarithmic derivatives to be quite robust to modeling uncertainty.

To explore the results shown in Figure 3 further, we show the posterior on the logarithmic slopes of $\rho_{g}^{\text {coll }}$ (top panel) and $\rho_{g}$ (bottom panel) evaluated at $R_{\text {sp }}$ in Figure 4 . We show the posteriors for model fits with $f_{\text {trans }}=$ free (red curves) and for $f_{\text {trans }}=1$ (green curves). Note, though, that since the $f_{\text {trans }}=1$ model is a special case of the $f_{\text {trans }}=$ free model and since we 
have shown that the latter model generally provides a better fit to the data, the $f_{\text {trans }}=$ free model should provide a more accurate representation estimate of the profile slope than the $f_{\text {trans }}=1$ model.

Figure 4 makes it clear that the data prefer a logarithmic slope of $\rho_{g}^{\text {coll }}$ at $R_{\mathrm{sp}}$ that is quite steep, indeed significantly steeper than the -3 expected for an NFW profile; the recovered estimate of the slope at $R_{\mathrm{sp}}$ is $-5.1 \pm 0.4$. The estimated slope of the total profile is also significantly steeper than -3 , with a recovered value of $-3.32 \pm 0.15$. Therefore, even allowing for considerable uncertainty in the profile of infalling matter, these results suggest that the profile of the collapsed component reaches slopes significantly steeper than -3 near $R_{\mathrm{sp}}$. Again, these findings can be taken as evidence for truncation of the halo profile similar to that seen in simulations by DK14. Unlike the model comparison results discussed in Section 4.1, these findings are quite robust to priors on the model parameters and choices of fitting scales.

Figure 4 also shows the results obtained from the model fits with $f_{\text {trans }}=1$ (green dashed curves). These fits yield significantly shallower slopes than the model fits, where $f_{\text {trans }}$ is allowed to vary. This may be surprising given that the logarithmic slopes of the projected profiles have almost identical steepness (see Figure 1). The explanation is that projection and miscentering act to smooth out a steep feature in the $3 \mathrm{D}$ profile, making it appear significantly less steep in the 2D profile. Even these fits, however, prefer slopes of $\rho_{g}^{\text {coll }}$ at $R_{\mathrm{sp}}^{3 \mathrm{D}}$ that are significantly steeper than -3 . Note, though, that $R_{\mathrm{sp}}^{3 \mathrm{D}}$ inferred from the $f_{\text {trans }}=1$ fits tends to be smaller than $R_{\mathrm{sp}}^{3 \mathrm{D}}$ inferred from the $f_{\text {trans }}=$ free fits (see Figure 1). Consequently, the distributions shown in Figure 4 are not coming from the same physical radius. Since the slope of the Einasto profile gets more negative with increasing radius, evaluating the $f_{\text {trans }}=$ free model slope at the $R_{\mathrm{sp}}^{3 \mathrm{D}}$ of the $f_{\text {trans }}=$ free fits would make the value of the slope more negative.

\subsection{Alternative Cluster Catalog}

We now consider the results of analyzing the measurements of $\Sigma_{g}$ around the groups identified in the Y07 catalog. As noted above, the use of an alternative cluster catalog provides an important systematics test for the existence of a splashback-like feature in the data.

Since the miscentering parameters appropriate for the Y07 groups are not known precisely, we use the wide miscentering priors of Model D in Table 1. However, given these wide priors and the lower signal-to-noise of the $\Sigma_{g}$ measurement for the Y07 groups, we find that for our fiducial analysis the model fits with $f_{\text {trans }}=$ free prefer values of $r_{t}$ that are at the edges of the prior on this parameter. We find that this can be prevented by imposing a somewhat tighter prior on $\alpha$ : $\log \alpha=\log (0.2) \pm 0.1$. This prior is still fairly loose relative to expectations from simulations, and is consistent with the values of $\alpha$ recovered from the analysis of REDMAPPER clusters when $f_{\text {trans }}$ is allowed to vary.

The $\Sigma_{g}$ measurements around the Y07 groups are shown in Figure 7 and the results for the model fits are summarized in Tables 1 and 2. In general, we find that the parameter values recovered from the Y07 fits agree quite well with those from the analysis of REDMAPPER clusters. The recovered splashback radius is also in good agreement. The Y07 analysis exhibits a large evidence ratio in support of the model with $f_{\text {trans }}=$ free, but this is somewhat misleading (at least in comparison to the REDMAPPER results) because of the prior we have imposed on $\alpha$ for this analysis.

Figure 8 shows the distribution of profile slopes for the Y07 groups, analogous to Figure 4 for the REDMAPPER clusters. The slope distributions shown in Figure 4 are quite consistent with those from the REDMAPPER measurement. They indicate logarithmic slopes of $\rho_{g}^{\text {coll }}$ at $R_{\text {sp }}$ of $-4.9 \pm 0.7$, significantly steeper than the slope expected for an NFW profile and consistent with expectations for a splashback feature.

We note that this test does not completely exclude the possibility of systematic effects introduced by the cluster finder (REDMAPPER or Y07), which could bias the location of $R_{\mathrm{sp}}$ or the steepness of the collapsed component at $R_{\mathrm{sp}}$. Nevertheless, the fact that both REDMAPPER and Y07 strongly prefer logarithmic slopes that are significantly steeper than -3 at $R_{\mathrm{sp}}$ is fairly convincing evidence that the finding of truncation of the halo profile is robust.

\section{Connecting the Halo Boundary to Galaxy Colors}

Our analysis has until now focused on examining the total galaxy density profile near the transition between the infall regime and the collapsed regime. Another approach to probing this transition is to examine galaxy colors. The passage of a galaxy through a cluster is expected to quench star formation in the galaxy. This process can happen through several channels: gravitational interactions with other galaxies or the cluster potential itself (e.g., Moore et al. 1996), stripping of the gas in the galaxy as a result of ram pressure from cluster gas (e.g., Gunn \& Gott 1972), and stripping of gas from the galaxy's gaseous halo, thereby preventing replenishment of gas used to form stars (i.e., "strangulation," see, e.g., Larson et al. 1980; Kawata \& Mulchaey 2008). Regardless of how it happens, quenching of star formation will cause a galaxy to appear redder than galaxies with active star formation. Measurements of a transition in galaxy clusters near the cluster virial radius have been performed in several previous studies including Dressler et al. (1997), Weinmann et al. (2006), and references therein. Here, we focus on the shape of this transition and its connection to the phase space boundary between the infalling and collapsed regimes.

In these scenarios, the typical timescales for quenching are comparable to the time taken to move across the extent of the cluster, roughly 2-4 Gyr (Wetzel et al. 2013). Therefore, if interactions within the cluster are responsible for quenching, a galaxy that has undergone a single passage through a cluster will appear redder than a galaxy that has not yet passed through the cluster. Since galaxies outside the splashback radius are significantly more likely to still be on their first infall, we expect a sharp increase in the fraction of red galaxies near the splashback radius.

Another possibility is that galaxy color simply correlates with formation time or the time of accretion of the galaxy onto the cluster and is not affected by processes inside the cluster (e.g., Hearin et al. 2015). In this case, a sharp increase in the red fraction at $R_{\mathrm{sp}}^{3 \mathrm{D}}$ would still be expected because within this radius the infalling galaxies suddenly start to be mixed with galaxies that were accreted 2-4 Gyr ago, which would also result in a sharp change in the red fraction. The main point in 

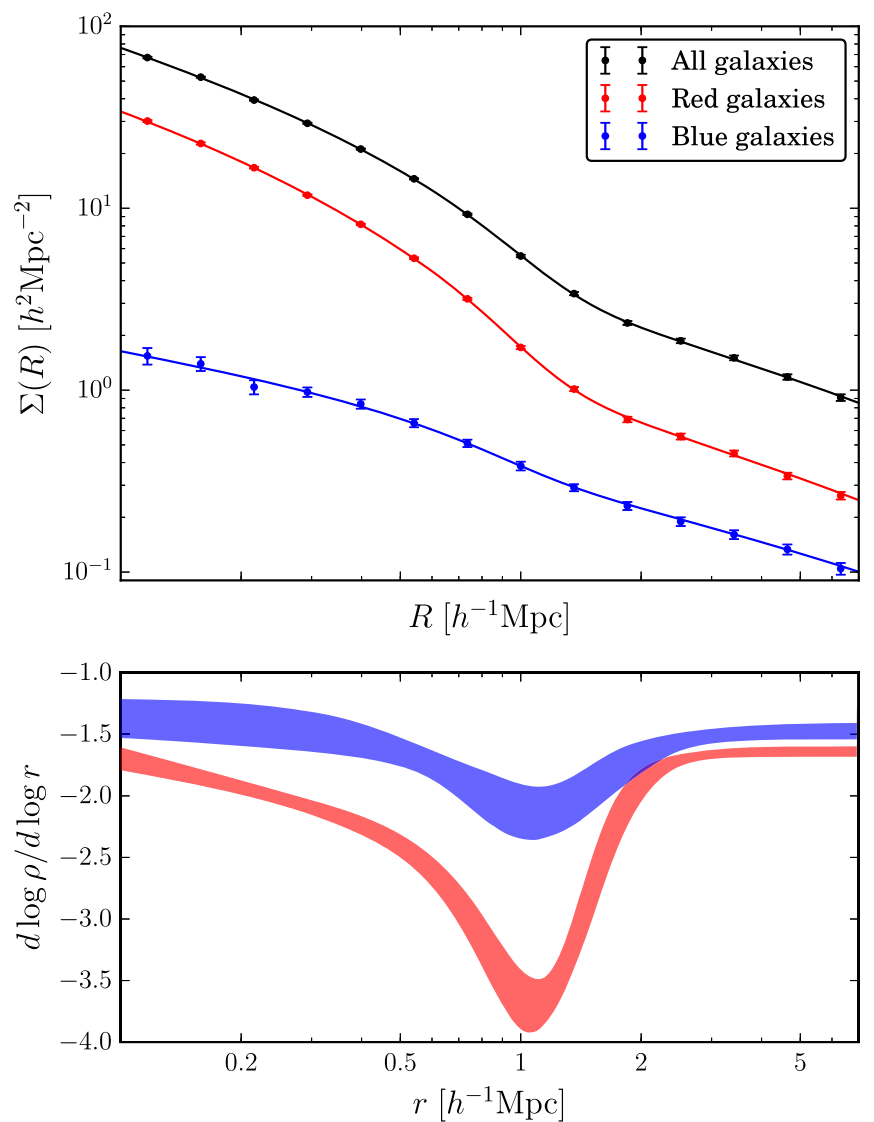

Figure 5. Top panel shows the $\Sigma_{g}(R)$ measurements for the full sample (black data points), the reddest quartile of galaxies (red data points) and bluest quartile of galaxies (blue points); best-fit models to the different measurements are shown as solid lines. The bottom panel shows the corresponding logderivatives of $\rho_{g}(r)$ inferred from model fiting.

the context of this paper is that in both scenarios the sharp increase in the red fraction is associated with the transition from the infalling to collapsed regimes at the splashback radius.

To investigate this, we consider two galaxy subsamples selected based on their rest-frame colors as described in Section 2. We measure the galaxy density profiles of the individual subsamples, and we will define the red/blue fraction to be the ratio of these two profiles to the $\Sigma_{g}$ for the full galaxy sample. The $\Sigma_{g}$ and corresponding model fits for the two galaxy color subsamples and the full sample are shown in the top panel of Figure 5; the inferred logarithmic derivatives of the 3D profile from the model fits are shown in the bottom panel. For this analysis, we use the Model $\mathrm{C}$ miscentering priors because these reflect recent constraints from analysis of REDMAPPER clusters.

Note that the profile of the blue galaxies approaches a power law with index close to -1.5 at large scales. Such a power law is precisely the expectation for infalling material that has not reached shell crossing (Dalal et al. 2010). Consider particles outside of $R_{\mathrm{sp}}^{3 \mathrm{D}}$ that are falling toward a cluster that dominates the local mass distribution. In the absence of shell crossing, the mass interior to the particles remains constant as they fall, and so their free-fall velocity scales as $v \sim r^{-0.5}$, where $r$ is the distance between the cluster center and the particles. The mass contributed by such particles to a radial shell at $r$ and with

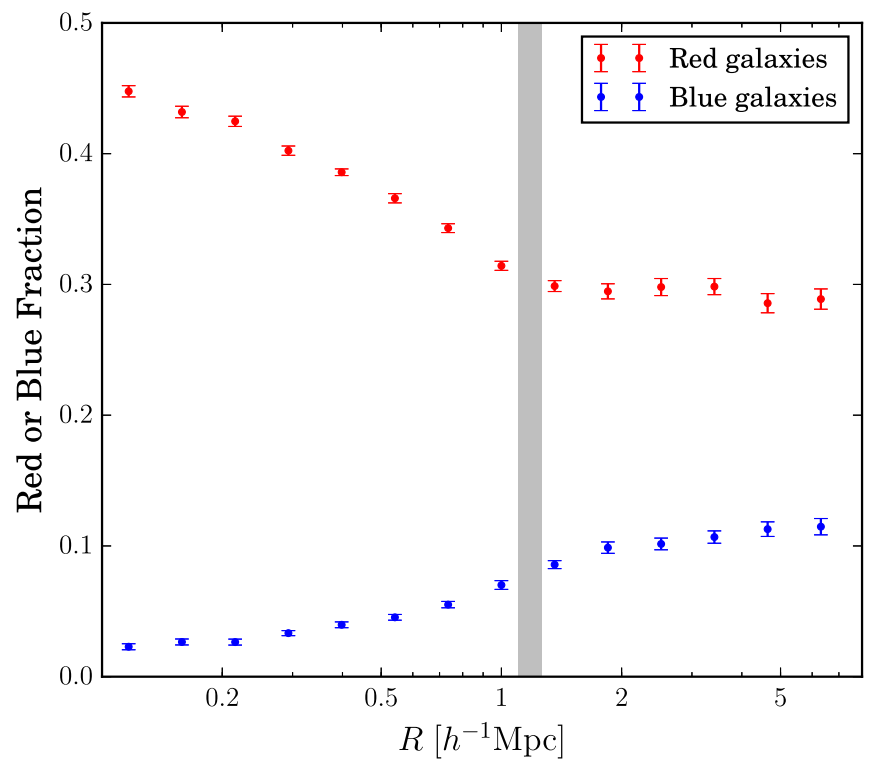

Figure 6. Fraction of red and blue galaxies relative to all galaxies around REDMAPPER clusters as a function of the projected distance from the cluster center.

thickness $d r$ will be proportional to the time the particles spend in the shell, so $(d M(r) / d r) d r \sim d r / v$, where $M(r)$ is the mass enclosed at radius $r$. Assuming $M(r)$ follows a power law and substituting the radial dependence of $v$, we have $M(r) \sim r^{1.5}$. The density profile, then, scales as $\rho \sim M / r^{3} \sim r^{-1.5}$.

The blue galaxy sample appears to be quite consistent with a purely infalling component at large $r$. In "pre-processing" models, quenching occurs in the dense environment surrounding the cluster, but prior to falling into the cluster (Fujita 2004). Quenching in this way should remove galaxies from the blue sample as $r$ decreases and add galaxies to the red sample, leading to a departure from the expected slope of -1.5 . The fact that we observe logarithmic derivatives close to -1.5 constrains the degree to which pre-processing contributes to quenching. Note, however, that we do observe slightly steeper slopes for the red galaxies than for the blue galaxies, which could be consistent with some amount of quenching due to preprocessing. This picture is complicated somewhat by the presence of so-called "backsplash" galaxies that have passed through the cluster (and may therefore have been quenched by processes inside the cluster) but have been ejected as a result of gravitational slingshot to several virial radii (Wetzel et al. 2014). We postpone more in depth modeling of these scenarios to future work. One further caveat to this discussion is that the large-scale logarithmic derivative of $\rho(r)$ inferred from the model fits depends weakly on the limits of integration imposed in Equation (7), i.e., $h_{\max }$. When varying $h_{\max }$ from $20 h^{-1} \mathrm{Mpc}$ to $60 h^{-1} \mathrm{Mpc}$ we find that the large-scale logarithmic derivative changes by roughly $1 \sigma$. These changes, however, do not significantly impact the conclusions of the analysis in this section.

Next, we show the red/blue fraction measurements in Figure 6. It is clear from Figure 6 that the red fraction shows an abrupt steepening at around $1.2 h^{-1}$ Mpc. In a scenario without a phase space boundary between the infalling and collapsed regimes, it is hard to imagine how a sharp upturn in 
the red fraction could arise at such large scales. The gas densities at these radii are quite low; how would a galaxy passing through the cluster outskirts know to become red at this particular radius? The picture of phase space caustics and quenching by the cluster (or at some time after accretion onto the cluster) provide a natural explanation for the observed red fraction behavior. In this picture, the galaxy quenches after one or more passages through the cluster, and the transition from outside $R_{\mathrm{sp}}$ to inside $R_{\mathrm{sp}}$ marks the transition from a regime for which most galaxies have never undergone a passage through the cluster to a regime for which most galaxies have undergone passage through the cluster. In support of this picture, the location of the upturn in the red fraction is in excellent agreement with the $R_{\mathrm{sp}}^{3 \mathrm{D}}$ inferred from the galaxy density measurements (shown as the gray band in Figure 6). Note that the three-dimensional $R_{\mathrm{sp}}^{3 \mathrm{D}}$ is the relevant radius of comparison since it is this radius that marks the physical phase space boundary in projection. The agreement between the projected red fraction measurements and the 3D splashback radius is non-trivial.

\section{Discussion}

\subsection{Evidence for A Halo Boundary}

Secondary infall models have predicted caustics in the phase space distribution of particles being accreted onto halos since the early studies of Fillmore \& Goldreich (1984) and Bertschinger (1985). It was not clear, however, whether the disruptive processes in the formation of cold dark matter halos would smear out caustic-like features, especially when stacking across many halos. Recently, using simulations and analytic arguments, DK14 and Adhikari et al. (2014) identified a rapid steepening of the density profile of stacked halos in $N$-body simulations that they associated with a density caustic arising from the second turnaround of matter particles, also known as splashback. Recent work by M16 has presented evidence of a narrow steepening of the galaxy density around REDMAPPER clusters detected in SDSS; such a finding is consistent with expectations for a splashback feature.

In this work, we attempt to determine to what extent available data support the existence of a halo boundary related to the presence of a phase space boundary between infalling and collapsed contributions to the total density profile. Two analyses are presented here.

1. We decompose the profile into "infalling" and "collapased" (or one-halo) components and use a model fitting approach to estimate the slope of the collapsed component near the transition between these two regimes. Near the location where the steepest slope of the total profile occurs, the one-halo profile reaches a logarithmic slope of about -5 over a narrow range of radius. This is significantly steeper than the expectation of an NFWlike profile, and supports the idea of a truncated halo profile.

2. The second evidence is that the location of steepest slope of the total profile coincides with an abrupt increase in the fraction of red galaxies. Presumably a fraction of galaxies inside the halo are quenched due to one or more passages through the cluster. In the infalling regime, on the other hand, galaxies have (for the most part) never been inside the cluster and are therefore likely to have at most a gradual trend in red fraction.

The results of these two analyses, shown in Figures 3 and 6, lend support to the presence of a phase space halo boundary associated with a sharp decline in the halo density profile. In contrast with common definitions of halo boundaries, such as $R_{200}$ or $R_{\text {vir }}$, a phase space halo boundary at $R_{\mathrm{sp}}$ would constitute a real, physical boundary to the halo (More et al. 2015).

As a systematics test of these findings, we repeat the measurements of galaxy density around groups identified in the Y07 catalog. We find that these measurements prefer similar slopes of the halo profile in the infalling-to-collapsed transition region as the REDMAPPER clusters, as shown in Figure 8. Recent work by $\mathrm{Zu}$ et al. (2016) found that the $\left\langle R_{\mathrm{mem}}\right\rangle$ quantity used by M16 to split their cluster sample can be severely impact by projections along the line of sight, which are in turn related to the local environment of the cluster. Since M16 found large changes in the inferred splashback radius with $\left\langle R_{\text {mem }}\right\rangle$, one may worry about the effects of projections on the measurement of the splashback feature. Our analysis does not preclude the possibility that $R_{\mathrm{sp}}$ is affected by projection effects or by selection effects inherent to REDMAPPER. However, while projections might smooth out an otherwise sharp splashback feature or change the inferred $R_{\text {sp }}$, it seems unlikely that they could be responsible for the artificial appearance of a splashback-like steepening. On the other hand, it is possible that some feature of the REDMAPPER algorithm could cause clusters that exhibit a sharp decline in their profiles to be preferentially selected, thereby leading to the appearance of a splashback feature. Our measurement of a rapid steepening around the Y07 groups, however, disfavors the possibility of the observed steepening being due solely to a REDMAPPER artifact. Since every cluster finder is, in principle, susceptible to spatially dependent selection effects, such a test cannot be definitive. However, extending the measurements performed here to cluster samples selected based on e.g., Sunyaev Zel'dovich decrement would provide an independent check on the optical cluster samples used in this work.

Using the parameterized models for the splashback feature introduced by DK14, we have also attempted to quantify whether the data support a model that has such a feature over a model that does not. We perform a model comparison by computing a Bayesian odds ratio between a model that includes the steepening caused by a splashback feature (parameterized via $f_{\text {trans }}$ in Equation (4)) and a pure Einasto profile that does not have additional steepening. A similar approach was taken by M16 to quantify the significance of their splashback detection, though only $\chi^{2}$ was reported there. We extend the modeling efforts of M16 to include miscentering, an important systematic affecting the galaxy density profile. We find, however, that given reasonably weak priors on the parameters of the pure Einasto model, this model can come close to matching the steepening of the model with additional steepening caused by splashback. We therefore conclude that until better data are available or tighter priors on the model parameters can be obtained, such a test is not a particularly useful way to quantify evidence for a splashbacklike feature in the data.

While basic uncertainties remain in connecting observations of a projected density profile (further modulated by the relation between galaxies and mass) with a boundary in phase space, 
we believe the findings summarized above are a promising indication of a dynamically motivated halo boundary. They provide avenues for studying physical processes such as dynamical friction (Adhikari et al. 2016), dark energy (Stark et al. 2016), self-interacting dark matter, and modifications of gravity (M16).

\subsection{Future Prospects}

In this analysis, we have used the galaxy density profile and the red fraction in an attempt to infer something about the phase space behavior near the boundaries of cluster halos. There may be alternate routes to probing this boundary that go beyond these two observables. For instance, proxies for halo accretion rate would be of significant utility to studies of splashback since the accretion rate is expected to correlate with the location of the splashback feature. While the analysis of M16 attempted to use $\left\langle R_{\text {mem }}\right\rangle$ as a proxy for accretion, subsequent work by $\mathrm{Zu}$ et al. (2016) has shown that this proxy is contaminated by projection effects. Another avenue, namely the impact of dynamical friction on splashback, has been explored by Adhikari et al. (2016) and would be valuable to test with future data sets. Our measurement of the red fraction provides qualitative evidence for a phase space boundary. However, more definitive statements about how the red fraction relates to splashback will likely require further effort with models and simulations of galaxies in cluster environments. The present work motivates exploration of these various avenues for detecting the splashback feature.

Ongoing and future galaxy surveys such as the Hyper SuprimeCam ${ }^{17}$ (HSC), the Dark Energy Survey ${ }^{18}$ (DES), the Kilo Degree Survey ${ }^{19}$ (KiDS), and the Large Synoptic Survey Telescope $^{20}$ (LSST) will provide much higher statistical power to constrain splashback models than the SDSS data. Together with better understanding of priors for both the model of the galaxy profile and systematic effects such as miscentering, we should expect significant improvement in our ability to characterize the splashback feature in the near future. The use of weak lensing measurements to more directly measure the cluster mass profile and study splashback (e.g., Umetsu \& Diemer 2017) may also be fruitful, particularly with upcoming weak lensing data sets from DES, HSC, and KiDS.

Finally, we note that this work has not addressed in any detail the location of the splashback feature. Although our model fits differ from those of M16, we do not find any significant difference in the recovered values of $R_{\mathrm{sp}}$ (though we have not performed any splits on $\left\langle R_{\mathrm{mem}}\right\rangle$ in this analysis). More exploration into the location of $R_{\mathrm{sp}}$ and comparison to simulations is a fruitful avenue for future work.

We thank Kathleen Eckert, William Hartley, Rachel Mandelbaum, Philip Mansfield, Carles Sánchez, Yao-Yuan Mao, Risa Wechsler, Kathy Romer, Tesla Jeltema, Simon White, and Ying $\mathrm{Zu}$ for fruitful discussions while preparing this manuscript. We also thank collaborators in the Dark Energy Survey for useful input to the analysis. C.C. and A.K. were supported in part by the Kavli Institute for Cosmological Physics at the University of Chicago through grant NSF PHY-

\footnotetext{
17 http://www.naoj.org/Projects/HSC

18 http://www.darkenergysurvey.org

19 kids.strw.leidenuniv.nl

${ }^{20}$ http://www.lsst.org
}

Table 3

Additional Best-fit Model Parameters with $f_{\text {trans }}$ Free (Number Preceding Semicolon in Each Column) and $f_{\text {trans }}=1$ (Number Following Semicolon) and Under Different Modeling Assumptions

\begin{tabular}{lcccc}
\hline \hline Model & Catalog & $\rho_{s}\left[h^{3} \mathrm{Mpc}^{-3}\right]$ & $\rho_{0}\left[h^{3} \mathrm{Mpc}^{-3}\right]$ & $s_{e}$ \\
\hline A & RM & $3.76 ; 22.58$ & $0.43 ; 0.27$ & $1.61 ; 1.34$ \\
B & RM & $27.08 ; 37.15$ & $0.43 ; 0.31$ & $1.61 ; 1.43$ \\
C & RM & $36.99 ; 103.92$ & $0.43 ; 0.39$ & $1.61 ; 1.55$ \\
D & RM & $48.98 ; 121.73$ & $0.43 ; 0.41$ & $1.61 ; 1.58$ \\
E & RM & $23.35 ; 14.86$ & $0.43 ; 0.43$ & $1.61 ; 1.60$ \\
F & RM & $11.72 ; 2043.12$ & $0.43 ; 0.12$ & $1.61 ; 1.05$ \\
\hline G & Y07 & $15.75 ; 29.53$ & $0.32 ; 0.21$ & $1.63 ; 1.41$ \\
\hline
\end{tabular}

1125897 and an endowment from Kavli Foundation and its founder Fred Kavli. E.B. and B.J. are partially supported by the US Department of Energy grant DE-SC0007901. E.R. is supported in part by DOE grant DE-SC0015975 and Sloan Foundation grant FG-2016-6443.

Funding for the Sloan Digital Sky Survey IV has been provided by the Alfred P. Sloan Foundation, the U.S. Department of Energy Office of Science, and the Participating Institutions. SDSS-IV acknowledges support and resources from the Center for High-Performance Computing at the University of Utah. The SDSS web site is http://www.sdss.org.

SDSS-IV is managed by the Astrophysical Research Consortium for the Participating Institutions of the SDSS Collaboration including the Brazilian Participation Group, the Carnegie Institution for Science, Carnegie Mellon University, the Chilean Participation Group, the French Participation Group, HarvardSmithsonian Center for Astrophysics, Instituto de Astrofísica de Canarias, The Johns Hopkins University, Kavli Institute for the Physics and Mathematics of the Universe (IPMU)/University of Tokyo, Lawrence Berkeley National Laboratory, Leibniz Institut für Astrophysik Potsdam (AIP), Max-Planck-Institut für Astronomie (MPIA Heidelberg), Max-Planck-Institut für Astrophysik (MPA Garching), Max-Planck-Institut für Extraterrestrische Physik (MPE), National Astronomical Observatories of China, New Mexico State University, New York University, University of Notre Dame, Observatário Nacional/MCTI, The Ohio State University, Pennsylvania State University, Shanghai Astronomical Observatory, United Kingdom Participation Group, Universidad Nacional Autónoma de México, University of Arizona, University of Colorado Boulder, University of Oxford, University of Portsmouth, University of Utah, University of Virginia, University of Washington, University of Wisconsin, Vanderbilt University, and Yale University.

\section{Appendix A Model Parameter Constraints}

Table 3 shows the fit results for the remaining parameters not shown in Table 2.

Appendix B

\section{Galaxy Density Measurement around Y07 Groups}

The measurement of galaxy density around the Y07 groups is shown in Figure 7. The distribution of logarithmic slopes at $R_{\mathrm{sp}}$ corresponding to this measurement is shown in Figure 8. 


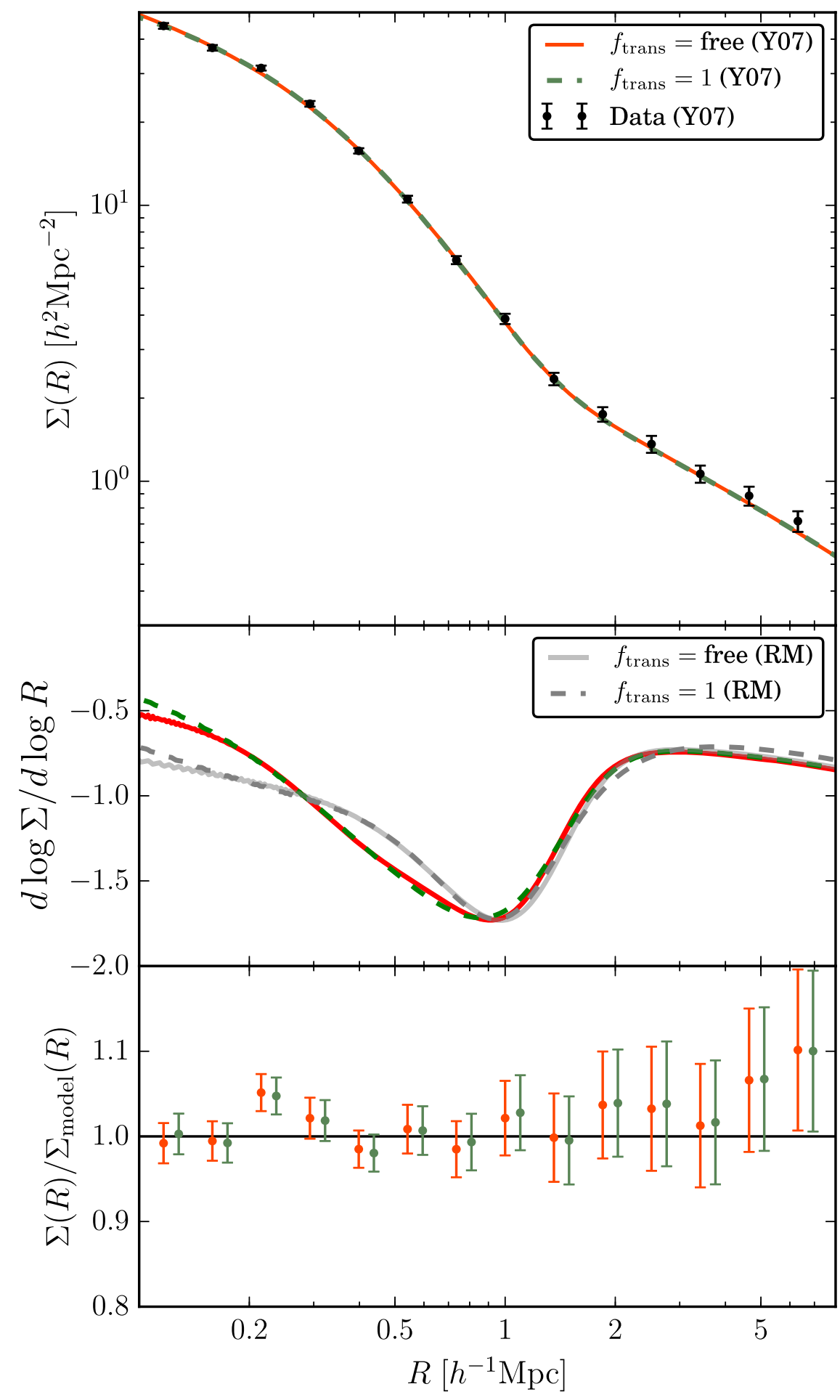

Figure 7. Measurement of the splashback feature using the Y07 group catalog. The top panel shows the projected galaxy density profile $\Sigma_{g}$ overlaid with models with $f_{\text {trans }}$ free (red solid) and $f_{\text {trans }}=1$ (green dashed). The middle panel shows the log-derivative of $\Sigma_{g}$. We note that the two model fits are nearly identical in both panels. We also overlay in gray the same measurements shown in Figure 1, which is based on the REDMAPPER (RM) cluster catalog. The feature around $1 h^{-1}$ Mpc in the REDMAPPER measurements appear slightly sharper than the Y07 group measurement. The bottom panel shows the ratio of the Y07 measurements to the best-fit models. 

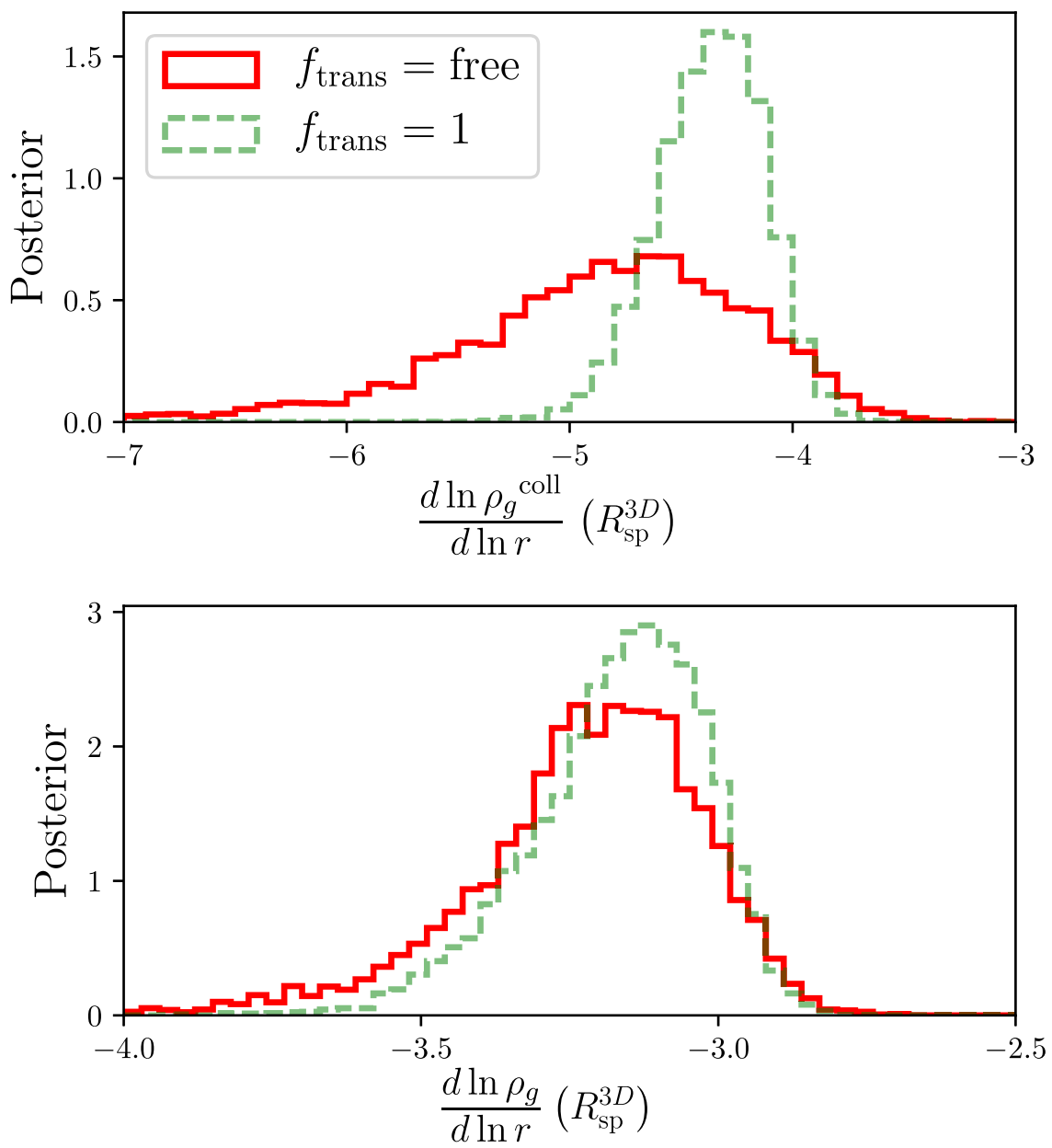

Figure 8. Same as Figure 4, but for the measurement of galaxy density around the groups in the Y07 catalog.

\section{References}

Abbas, U., \& Sheth, R. K. 2007, MNRAS, 378, 641

Adhikari, S., Dalal, N., \& Chamberlain, R. T. 2014, JCAP, 11, 019

Adhikari, S., Dalal, N., \& Clampitt, J. 2016, JCAP, 7, 022

Aihara, H., Allende Prieto, C., An, D., et al. 2011, ApJS, 193, 29

Behroozi, P. S., Wechsler, R. H., \& Wu, H.-Y. 2013, ApJ, 762, 109

Bertschinger, E. 1985, ApJS, 58, 39

Bryan, G. L., \& Norman, M. L. 1998, ApJ, 495, 80

Budzynski, J. M., Koposov, S. E., McCarthy, I. G., McGee, S. L., \& Belokurov, V. 2012, MNRAS, 423, 104

Busha, M. T., Evrard, A. E., Adams, F. C., \& Wechsler, R. H. 2005, MNRAS, 363, L11

Cavagnolo, K. W., Donahue, M., Voit, G. M., \& Sun, M. 2009, ApJS, 182, 12

Cooray, A., \& Sheth, R. 2002, PhR, 372, 1

Dalal, N., Lithwick, Y., \& Kuhlen, M. 2010, arXiv:1010.2539

Diemer, B., \& Kravtsov, A. V. 2014, ApJ, 789, 1

Dressler, A., Oemler, A., Jr., Couch, W. J., et al. 1997, ApJ, 490, 577

Dutton, A. A., \& Macciò, A. V. 2014, MNRAS, 441, 3359

Einasto, J. 1965, TrAlm, 5, 87

Fillmore, J. A., \& Goldreich, P. 1984, ApJ, 281, 1

Foreman-Mackey, D., Hogg, D. W., Lang, D., \& Goodman, J. 2013, PASP, 125,306

Fujita, Y. 2004, PASJ, 56, 29

Gao, L., Navarro, J. F., Cole, S., et al. 2008, MNRAS, 387, 536

George, M. R., Leauthaud, A., Bundy, K., et al. 2012, ApJ, 757, 2

Gifford, D., Kern, N., \& Miller, C. J. 2017, ApJ, 834, 204

Gunn, J. E., \& Gott, J. R., III 1972, ApJ, 176, 1

Guo, Q., White, S., Boylan-Kolchin, M., et al. 2011, MNRAS, 413, 101

Hearin, A. P., Watson, D. F., \& van den Bosch, F. C. 2015, MNRAS, 452, 1958

Hernquist, L. 1990, ApJ, 356, 359
Hoshino, H., Leauthaud, A., Lackner, C., et al. 2015, MNRAS, 452, 998 Ivezić, Ž., Connelly, A. J., VanderPlas, J. T., \& Gray, A. 2014, Statistics, Data Mining, and Machine Learningin Astronomy (Princeton, NJ: Univ of Princeton)

Jeffreys, H., \& Lindsay, R. B. 1963, PhT, 16, 68

Johnston, D. E., Sheldon, E. S., Wechsler, R. H., et al. 2007, arXiv:0709.1159

Kawata, D., \& Mulchaey, J. S. 2008, ApJL, 672, L103

Landy, S. D., \& Szalay, A. S. 1993, ApJ, 412, 64

Larson, R. B., Tinsley, B. M., \& Caldwell, C. N. 1980, ApJ, 237, 692

Mansfield, P., Kravtsov, A. V., \& Diemer, B. 2016, arXiv:1612.01531

Masjedi, M., Hogg, D. W., Cool, R. J., et al. 2006, ApJ, 644, 54

Mehrtens, N., Romer, A. K., Hilton, M., et al. 2012, MNRAS, 423, 1024

Melchior, P., Gruen, D., McClintock, T., et al. 2016, arXiv:1610.06890

Melchior, P., Suchyta, E., Huff, E., et al. 2015, MNRAS, 449, 2219

Merritt, D., Graham, A. W., Moore, B., Diemand, J., \& Terzić, B. 2006, AJ, 132, 2685

Merritt, D., Navarro, J. F., Ludlow, A., \& Jenkins, A. 2005, ApJL, 624, L85

Miyatake, H., More, S., Takada, M., et al. 2016, PhRvL, 116, 041301

Moore, B., Katz, N., Lake, G., Dressler, A., \& Oemler, A. 1996, Natur, 379,613

More, S., Diemer, B., \& Kravtsov, A. V. 2015, ApJ, 810, 36

More, S., Miyatake, H., Takada, M., et al. 2016, ApJ, 825, 39

Nagai, D., \& Kravtsov, A. V. 2005, ApJ, 618, 557

Navarro, J. F., Frenk, C. S., \& White, S. D. M. 1996, ApJ, 462, 563

Navarro, J. F., Hayashi, E., Power, C., et al. 2004, MNRAS, 349, 1039

Navarro, J. F., Ludlow, A., Springel, V., et al. 2010, MNRAS, 402, 21

Peacock, J. A., \& Smith, R. E. 2000, MNRAS, 318, 1144

Rines, K., Geller, M. J., Diaferio, A., \& Kurtz, M. J. 2013, ApJ, 767, 15

Rozo, E., \& Rykoff, E. S. 2014, ApJ, 783, 80

Rykoff, E. S., Rozo, E., Busha, M. T., et al. 2014, ApJ, 785, 104

Seljak, U. 2000, MNRAS, 318, 203

Sérsic, J. L. 1963, BAAA, 6, 41

Sheldon, E. S., Johnston, D. E., Masjedi, M., et al. 2009, ApJ, 703, 2232 
Sheth, R. K., Hui, L., Diaferio, A., \& Scoccimarro, R. 2001, MNRAS, 325,1288

Simet, M., McClintock, T., Mandelbaum, R., et al. 2017, MNRAS, 466, 3103

Soares-Santos, M., de Carvalho, R. R., Annis, J., et al. 2011, ApJ, 727, 45

Stark, A., Miller, C. J., \& Huterer, D. 2016, arXiv:1611.06886

Tully, R. B. 2015, AJ, 149, 54

Umetsu, K., \& Diemer, B. 2017, ApJ, 836, 231

van Breukelen, C., \& Clewley, L. 2009, MNRAS, 395, 1845

van Uitert, E., Cacciato, M., Hoekstra, H., \& Herbonnet, R. 2015, A\&A, 579, A26

Weinmann, S. M., van den Bosch, F. C., Yang, X., \& Mo, H. J. 2006, MNRAS, 366, 2
Wen, Z. L., Han, J. L., \& Liu, F. S. 2012, ApJS, 199, 34

Wetzel, A. R., Tinker, J. L., Conroy, C., \& van den Bosch, F. C. 2013, MNRAS, 432, 336

Wetzel, A. R., Tinker, J. L., Conroy, C., \& van den Bosch, F. C. 2014, MNRAS, 439, 2687

Yang, X., Mo, H. J., van den Bosch, F. C., \& Jing, Y. P. 2005, MNRAS, 356,1293

Yang, X., Mo, H. J., van den Bosch, F. C., et al. 2006, MNRAS, 373 1159

Yang, X., Mo, H. J., van den Bosch, F. C., et al. 2007, ApJ, 671, 153

Zu, Y., Mandelbaum, R., Simet, M., Rozo, E., \& Rykoff, E. S. 2016, arXiv:1611.00366 